Transfer Learning for Unsupervised Influenza-like Illness Models from Online Search Data

Zou, Bin; Lampos, Vasileios; Cox, Ingemar

Published in:

WWW '19: The World Wide Web Conference

DOI:

$10.1145 / 3308558.3313477$

Publication date:

2019

Document version

Publisher's PDF, also known as Version of record

Document license:

CC BY

Citation for published version (APA):

Zou, B., Lampos, V., \& Cox, I. (2019). Transfer Learning for Unsupervised Influenza-like Illness Models from Online Search Data. In WWW'19: The World Wide Web Conference (pp. 2505-2516). Association for Computing Machinery. https://doi.org/10.1145/3308558.3313477 


\section{Transfer Learning for Unsupervised Influenza-like Illness Models from Online Search Data}

\author{
Bin Zou \\ Department of Computer Science \\ University College London \\ United Kingdom \\ bin.zou.14@ucl.ac.uk
}

\author{
Vasileios Lampos \\ Department of Computer Science \\ University College London \\ United Kingdom \\ v.lampos@ucl.ac.uk
}

\author{
Ingemar J. Cox \\ Department of Computer Science \\ University College London \\ United Kingdom \\ i.cox@ucl.ac.uk
}

\begin{abstract}
A considerable body of research has demonstrated that online search data can be used to complement current syndromic surveillance systems. The vast majority of previous work proposes solutions that are based on supervised learning paradigms, in which historical disease rates are required for training a model. However, for many geographical regions this information is either sparse or not available due to a poor health infrastructure. It is these regions that have the most to benefit from inferring population health statistics from online user search activity. To address this issue, we propose a statistical framework in which we first learn a supervised model for a region with adequate historical disease rates, and then transfer it to a target region, where no syndromic surveillance data exists. This transfer learning solution consists of three steps: (i) learn a regularized regression model for a source country, (ii) map the source queries to target ones using semantic and temporal similarity metrics, and (iii) re-adjust the weights of the target queries. It is evaluated on the task of estimating influenza-like illness (ILI) rates. We learn a source model for the United States, and subsequently transfer it to three other countries, namely France, Spain and Australia. Overall, the transferred (unsupervised) models achieve strong performance in terms of Pearson correlation with the ground truth ( $>.92$ on average), and their mean absolute error does not deviate greatly from a fully supervised baseline.
\end{abstract}

\section{ACM Reference Format:}

Bin Zou, Vasileios Lampos, and Ingemar J. Cox. 2019. Transfer Learning for Unsupervised Influenza-like Illness Models from Online Search Data. In Proceedings of the 2019 World Wide Web Conference (WWW'19), May 13-17, 2019, San Francisco, CA, USA. ACM, New York, NY, USA, 12 pages. https://doi.org/10.1145/3308558.3313477

\section{INTRODUCTION}

Syndromic surveillance systems aim to provide timely estimates about the prevalence of a disease in a population. Their main source of information is based on doctor assessments about the probable health status of patients given a set of symptoms. For example, to monitor the rate of influenza, syndromic surveillance relies on a

\footnotetext{
*Also affiliated with the University of Copenhagen, Denmark.
}

This paper is published under the Creative Commons Attribution 4.0 International (CC-BY 4.0) license. Authors reserve their rights to disseminate the work on their personal and corporate Web sites with the appropriate attribution.

WWW'19, May 13-17, 2019, San Francisco, CA, USA

() 2019 IW3C2 (International World Wide Web Conference Committee), published under Creative Commons CC-BY 4.0 License.

ACM ISBN 978-1-4503-6674-8/19/05.

https://doi.org/10.1145/3308558.3313477 network of doctors who report on a daily or weekly basis the number of patients exhibiting related symptoms, such as fever, cough or a sore throat. Recent research efforts have shown that this traditional approach can be complemented by alternative methods trained on data from online user activity, e.g. social media or online search behavior [43]. Applications vary from modelling dengue fever [24] to depression [17], but particular research focus has been drawn to influenza, an infectious disease that is responsible for 290-650,000 deaths worldwide on an annual basis. ${ }^{1}$ Data from the microblogging platform of Twitter $[15,32,50]$ as well as from search engines $[21,35,53,67]$ combined with statistical natural language processing methods have produced promising outcomes, which in some occasions have been incorporated into national influenza surveillance schemes $[9,63]$. The main advantages of these complementary methods are timeliness, and sampling from a larger segment of the population, including people who may not visit a doctor while being ill. It is also commonly cited that such approaches may be very useful in regions where health infrastructure is poor or absent. However, this is often impractical as the proposed machine learning solutions rely on training data which apart from the user-generated inputs, need to contain confirmed disease rates at the target location, broadly referred to as "ground truth". This data is typically provided by existing syndromic surveillance systems. Hence, for locations where ground truth is not available, user-data driven approaches are not realistically applicable.

In this paper, we propose a statistical framework to circumvent problems associated with no training data in some geographic regions. Our approach is based on the broad notion of transfer learning, where we aim to transfer parts of the knowledge gained while solving a certain task to better solve a different, but related one [49]. In particular, our goal is to transfer a well-performing disease rate inference model from a source location, where supervised learning is possible, to a target location, where supervision is not possible, given the lack of ground truth. We focus our experiments on influenza (flu) and utilize Google search query statistics as our descriptive variable for aggregate, population-level, online user activity. For example, the US Centers for Disease Control and Prevention (CDC) monitor and report influenza-like illness (ILI) rates on a weekly basis, providing sufficient ground truth to learn a function that maps online search query frequencies to these rates. In our experiments we show that we can adapt this function to derive estimates of ILI rates at different locations (outside the US). Language may or may not differ between the source and target locations. Online search statistics can be obtained for these target locations, but we assume that there is no ground truth data.

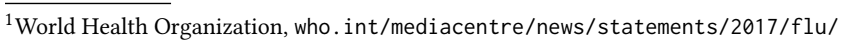


The proposed approach comprises 3 steps. After learning a source regression model (step 1), we seek ways to map the selected source search queries to sets of queries in the target location. To derive this mapping we deploy a hybrid metric, which combines a semantic similarity with a time series correlation component (step 2). Semantic similarities are estimated using cross-lingual or monolingual word embeddings and correlations are computed using query frequencies. Finally, query weights from the source model are transferred to the identified target queries (step 3). This framework is evaluated on three transfer learning tasks, where the source model is always based in the US, and the target countries are France, Spain and Australia. While ground truth is available for all the target countries, we only use it to evaluate the performance of the transferred models. Transferred models, assessed on four flu seasons (2012 to 2016), can accurately estimate the peak of each flu season, achieving on average Pearson correlations greater than .92 and root mean squared errors comparable to the ones obtained by the corresponding fully supervised models $(\leq 21.6 \%$ increase in errors). Therefore, they can be considered as practical solutions for locations that lack historical ground truth data.

Main contributions. A novel, end-to-end transfer learning framework is proposed for mapping a disease model trained on online search data from a location, where ground truth is available, to a location, where ground truth is not available. Variations of this model are investigated, exploring different query mapping functions using semantic or temporal similarities or combinations of the two. In addition, we empirically show that our approach works in three case studies, two of which require a transfer to a different language (English to French or Spanish), and one that maintains the same language (English), but demands a model transfer to a different hemisphere (US to Australia).

\section{DATA SETS}

We use two sources of data, namely Google search query frequency statistics and ILI rates from established health organizations.

Google search query frequency statistics. Time series of weekly search query frequencies were retrieved through Google Correlate. A frequency represents the weekly search activity of a query (number of times issued) within a geographical region. It is normalized by dividing by the total number of search queries issued during that week. This normalization controls for variations in the number of searches issued each week which can be due to a variety of causes, including summer vacations, responses to news events, and a longer-term trend of increased web usage [45]. Normalized query frequencies are subsequently standardized, such that their time series have a zero mean and a standard deviation of one. This results in expressing query frequencies under the same units for different geographical regions with potentially varying population sizes and search usage patterns. We obtained weekly frequencies of search queries from September 1, 2007 to August 31, 2016 inclusive (470 weeks) for US, France, Spain, and Australia. Given that an exhaustive list of user search queries was not available to us, we extracted them by first using a set of 12 flu-related queries per country as a seed to Google Correlate and then iterating through this process (using correlated queries as new seeds). This process extracted 34,121, 29,996, 15,673 and 8,764 queries for US, France,
Spain and Australia, respectively. Queries were not limited to the topic of flu, given that various other spurious queries may also correlate with the seeds.

ILI rates. We obtained weekly ILI rates for the US, France, Spain and Australia from their established syndromic surveillance systems, namely the Centers for Disease Control and Prevention (CDC), GPs Sentinelles Network (SN), Spanish Influenza Sentinel Surveillance System (SISSS), and Australian Sentinel Practices Research Network (ASPREN), respectively. ${ }^{2}$ ILI rates represent fraction of the population that has been diagnosed with influenza-like symptoms. ${ }^{3}$ The data spans from September 1, 2007 to August 31, 2016 inclusive, which covers approximately 9 consecutive influenza seasons. Note that for Spain, we only have ILI rates from week 40 in a year to week 20 in the following year. The prevalence of influenza outside this period is typically very low. ${ }^{4}$ We denote the ILI rates from each syndromic surveillance system using the corresponding country code (US, FR, ES, and AU).

In our experiments we are transferring a flu model trained on US data to one of the other three countries. To provide some insight about the difficulty of the task, we have plotted the historical ILI rates for all countries in Fig. 1. ILI rates may correlate between countries, e.g. the Pearson correlation between the US and FR rates is equal to $.6\left(p \approx 3 \cdot 10^{-54}\right)$, but peaks and troughs are occurring at different times and with very different intensity. The US and AU ILI rates are negatively correlated $\left(-.4, p \approx 8 \cdot 10^{-17}\right)$, as expected, since these countries are situated in different hemispheres and influenza is strongly seasonal. The optimal correlation we can obtain by shifting the ILI rate time series is equal to .68 (US-ES). Notably, the metric for ILI may differ in the countries we considered in this paper. Therefore, in our experiments we are working with a standardized representation of ILI (z-score).

\section{METHODS}

Disease rate estimation from online search data is commonly formulated as a regression task [21,35]. The aim is to learn a function $f: \mathbf{X} \rightarrow \mathbf{y}$ that maps the input space of search query frequencies, $\mathrm{X} \in \mathbb{R}^{n \times s}$, to the target variable, $\mathrm{y} \in \mathbb{R}^{n}$, representing disease rates; $n$ denotes the number of samples and $s$ is the size of the feature space, i.e. the number of unique search queries we are considering. More specifically, X contains the time series of search query frequencies, and $\mathrm{y}$ represents a rate of disease diagnoses in a population (as reported by a health agency) at corresponding times. The time interval for computing the frequency of queries is often set to one week to match the frequency of syndromic surveillance reports.

Regression approaches require observations of the target variable y (ground truth) for training a machine learning model. This restricts the application of such techniques to areas where historical disease rates are available. We attempt to address this limitation by proposing a transfer learning methodology, that maps an existing disease model, $f: \mathbf{X} \rightarrow \mathbf{y}$, from a source location, where disease rates are available, to another location, where disease rates are not possible to obtain. We define the source domain as $\mathcal{D}_{\mathrm{S}}=\left\{\left(\mathbf{x}_{i}, y_{i}\right)\right\}$,

\footnotetext{
${ }^{2}$ Links: CDC (US), cdc .gov; SN (FR), websenti .u707. jussieu. fr/sentiweb; SISSS (ES), eng.isciii.es/ISCIII; ASPREN (AU), aspren.dmac.adelaide.edu.au ${ }^{3}$ ILI is defined as the presence of high fever plus cough or sore throat [11, 46]. ${ }^{4}$ In Figs. 1 and 2, we have set the missing ILI rates for ES to zero for visualization purposes. However, we are not using these rates to train or evaluate models for ES.
} 


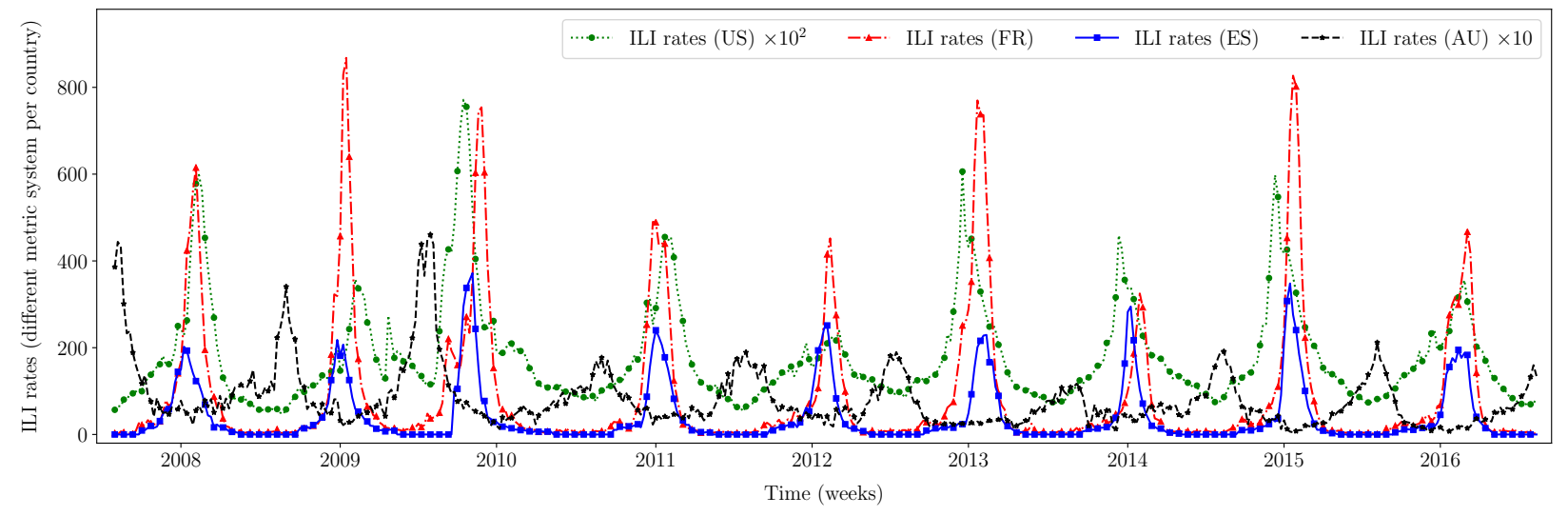

Figure 1: ILI rates for the United States (US), France (FR), Spain (ES) and Australia (AU).

$i \in\{1, \ldots, n\}$, where $\mathbf{x}_{i}$ is an $s$-dimensional vector holding the frequencies of the $s$ queries for the time interval $i, y_{i}$ is the corresponding disease rate, and $n$ is the number of observations. The target domain is denoted by $\mathcal{D}_{\mathrm{T}}=\left\{\mathbf{x}_{i}^{\prime}\right\}, i \in\{1, \ldots, m\}$, where $\mathbf{x}_{i}^{\prime}$ is a $t$-dimensional vector of the frequencies of the $t$ queries in the target domain that are going to be associated with the $s$ queries in the source domain. No ground truth is available for the target domain Note that $t$ need not equal $s$, thus allowing one-to-many query mappings. In theory, the $m$ time intervals may precede or overlap the $n$ time intervals in the source region. In our experiments, we the $m$ target intervals are always after the $n$ source intervals.

\subsection{User search behavior in different countries}

As the transfer learning framework is detailed in the next paragraphs, it will become apparent that it is grounded on a fundamental assumption, which is that online user search behavior will be similar in the source and the target countries. Narrowing this assumption down to our specific task, this implies that the conditional probability of issuing a query $q$ under a certain health status $h$ (with or without experiencing disease symptoms), $P(q \mid h)$, will be similar for the populations of the source and the target countries. Relevant literature offers some evidence about this with regards to user search behavior for various health-related themes $[1,3,25,68]$. In addition, we also provide some empirical evidence using our data. Table 1 shows the average query frequency over the corresponding ILI rate ratio for three basic queries in the US and AU. It also shows these ratios for translations of these queries in FR and ES (e.g. flu $\rightarrow$ grippe (FR) $\rightarrow$ gripe (ES)). The main observation is that these ratios do not vary much over the time span of our data, which is almost a decade. Although this is a limited observation, in that it does not involve many different search queries, it serves as a strong indication that user search behavior, at least for this specific area of interest, has similarities among different countries. The transfer learning framework, described in the following paragraphs, tries to exploit these similarities.

\subsection{Transfer learning framework}

The proposed transfer learning framework consists of three steps which are described in detail in the following sections.
3.2.1 Step 1 - Learning a regression function in the source domain. Regularized regression has been successfully applied to various text regression tasks, including the estimation of disease rates from social media or online search data [32, 35]. In this paper, we use elastic net [74] as our regression function, similarly to previous work on the topic $[35,37]$. Elastic net combines $\ell_{1}$-norm regularization, commonly known as the lasso [58], with $\ell_{2}$-norm, or ridge [26], regularization. In addition to the sparsity encouraged by the $\ell_{1}$ norm regularization, the $\ell_{2}$-norm regularizer attempts to address model consistency problems that arise when collinear predictors exist in the input space [69], which is common in text regression tasks $[34,36,54]$. Given $\mathrm{X} \in \mathbb{R}^{n \times s}$ and $\mathrm{y} \in \mathbb{R}^{n}$ from the source domain $\mathcal{D}_{S}$, we apply a constrained version of elastic net which solves the following optimization problem:

$$
\underset{\mathbf{w}, \beta}{\operatorname{argmin}}\left(\|\mathbf{y}-\mathbf{X w}-\beta\|_{2}^{2}+\lambda_{1}\|\mathbf{w}\|_{2}^{2}+\lambda_{2}\|\mathbf{w}\|_{1}\right) \text { subject to } \mathbf{w} \geq 0,
$$

where $\lambda_{1}>0, \lambda_{2}>0$ are respectively the $\ell_{1}$-norm and $\ell_{2}$-norm regularization parameters, and $\beta$ denotes the intercept term. The non-negativity constraint for $\mathbf{w}$ may result in a worse performing model for the source country, but, at the same time, makes the weight transfer from a source to a target country more comprehensible (positive weights are easier to interpret) and eventually more accurate in terms of performance (see Section 4.2).

Due to the seasonal nature of influenza, our dataset of candidate queries contains a significant number of confounders, i.e. queries with frequencies that are correlated to ILI rates, but have no link to flu, such as 'college basketball' or 'spring break'. To remove these unrelated queries we applied a semantic filter based on word embedding representations, similar to the one proposed in [38, 72, 73]. Word embeddings were trained on the English Wikipedia corpus using the fastText method [12]. A topic about flu, $\mathcal{T}$, was defined as a simple set of two flu-related terms, $\mathcal{T}=\{$ 'flu', 'fever' $\}$. For each of the source queries, we calculate a similarity score defined as the product of the cosine similarities between the embeddings of the terms in $\mathcal{T}$ and $\mathbf{e}_{\mathrm{q}}$, i.e.

$$
g(\mathbf{q}, \mathcal{T})=\cos \left(\mathbf{e}_{\mathrm{q}}, \mathbf{e}_{\mathcal{T}_{1}}\right) \times \cos \left(\mathbf{e}_{\mathrm{q}}, \mathbf{e}_{\mathcal{T}_{2}}\right),
$$

where each cosine similarity component is mapped to $[0,1]$ via $(\cos (\cdot, \cdot)+1) / 2 .{ }^{5}$ Queries from the source domain with $g \leq .5$ are

\footnotetext{
${ }^{5}$ This resolves misleading similarity scores based on different sign combinations.
} 
Table 1: Mean ratio of query frequency over ILI rate (and standard deviation of the mean) in four countries.

\begin{tabular}{|c|c|c|c|c|}
\hline Search queries & US & FR & ES & AU \\
\hline flu (US/AU), grippe (FR), gripe (ES) & $.036(.010)$ & $.033(.012)$ & $.032(.011)$ & $.031(.016)$ \\
\hline symptoms of flu (US/AU), symptômes de la grippe (FR), síntomas de gripe (ES) & $.030(.009)$ & $.031(.012)$ & $.029(.009)$ & $.027(.014)$ \\
\hline flu in children (US/AU), grippe chez le bébé (FR), gripe en el bebé (ES) & $.017(.007)$ & $.020(.008)$ & $.019(.009)$ & $.022(.010)$ \\
\hline
\end{tabular}

filtered out and are not considered in our experiments. The remaining queries are used to train an elastic net. This operation further reduces the selected queries to a subset $Q_{S}$, i.e. the ones that have been allocated a nonzero weight.

3.2.2 Step 2-Mapping source to target queries. The identified and weighted set of search queries in the source domain $\left(Q_{S}\right)$ should be mapped to a set of queries in the target domain from a potential pool of target query candidates $\left(\mathcal{P}_{\mathrm{T}}\right)$. Queries about the same topic may vary in their textual formulation, especially when they are issued by users located in different countries. Even in cases, where countries share the same language, cultural and socioeconomic differences may result into different querying preferences. Thus, simple approaches, where search queries from the source country are translated or directly mapped to queries in the target country, are not effective. ${ }^{6}$ In our approach, we utilize word embeddings (mono- or cross-lingual) to map source to target queries based on their broad semantic relationship. We consider both one-to-one and one-to-many query mappings from the source to the target domain. In addition, the weight associated with each source query reflects on how correlated the query is with the modeled disease rate. Therefore, another desired property is to map source queries to target ones based on their pairwise temporal correlation as this may enhance the statistical relevance of the mapping. Consequently, there is a trade-off between mapping based on semantic similarity and based on the similarity in temporal correlation. To capture both, we define a combined similarity metric, $\Theta$, that is the weighted sum of a semantic similarity $\Theta_{\mathrm{s}}$ and a correlation similarity, $\Theta_{\mathrm{c}}$, i.e.

$$
\Theta=\gamma \Theta_{\mathrm{s}}+(1-\gamma) \Theta_{\mathrm{c}},
$$

where $\gamma \in[0,1]$ controls the relative weighting of each. When $\gamma=1$ the mapping is based only on semantic similarity. Conversely, when $\gamma=0$ the mapping is based only on the correlation similarity.

Semantic similarity $\left(\Theta_{\mathrm{S}}\right)$. If the source and target domains have different languages, a translation module is required. For this purpose, we deploy cross-lingual word embeddings. Cross-lingual embeddings are trained using corpora from multiple languages, and can be used to compute word similarities in different languages [57, 60, 61]. Empirical evidence indicates that they can also facilitate better knowledge transfer between languages [2, 44, 47]. The majority of cross-lingual word embedding models are trained by exploiting sources of monolingual text alongside a smaller crosslingual corpus of aligned text [56]. The alignment can be made at word [2, 5, 18, 41, 57, 60], sentence [39, 75], and document level $[44,62]$. In this paper, we utilize a method for learning bilingual word embeddings proposed by Smith et al. [57].

First, for each of the source and target languages, we respectively learn a word embedding space based on monolingual text. For all languages considered in our experiments (English, French and

\footnotetext{
${ }^{6}$ We have empirical evidence about this, obtained during the first stages of this work
}

Spanish) we obtained word embeddings by applying fastText on corresponding Wikipedia corpora [12]. ${ }^{7}$ The dimensionality of the word embeddings was set to $d=300$. Then, we used a core selection of exact translation pairs $(\sigma \rightarrow \tau)$ from the source to the target domain language to generate bilingual embeddings. Given the embedding matrices of this alignment dictionary, $\mathbf{E}_{\sigma}$ and $\mathbf{E}_{\tau}$ both $\in \mathbb{R}^{m \times d}$, where $m, d$ denote the number of translation pairs and the dimensionality of the word embedding respectively, we learn a transformation matrix $\mathbf{W} \in \mathbb{R}^{d \times d}$ such that $\mathbf{E}_{\tau} \approx \mathbf{E}_{\sigma} \mathbf{W}$. W is an orthogonal matrix learned by minimizing the squared Euclidean distance between $\mathbf{E}_{\sigma}$ and $\mathbf{E}_{\tau}$, i.e.

$$
\underset{\mathbf{W}}{\operatorname{argmin}}\left\|\mathbf{E}_{\sigma} \mathbf{W}-\mathbf{E}_{\tau}\right\|_{2}^{2} \text {, subject to } \mathbf{W}^{\top} \mathbf{W}=\mathbf{I} \text {. }
$$

The orthogonality constraint ensures that the transformation works both ways, that is $\mathbf{E}_{\tau} \approx \mathbf{E}_{\sigma} \mathbf{W}, \mathbf{E}_{\sigma} \approx \mathbf{E}_{\tau} \mathbf{W}^{\top}$, and $\mathbf{E}_{\tau} \approx \mathbf{E}_{\tau} \mathbf{W}^{\top} \mathbf{W}$ [57]. In addition, Artexte et al. have empirically shown that it also improves the performance of machine translation [4]. The exact solution of Eq. 4 is given by $\mathbf{W}=\mathbf{V} \mathbf{U}^{\top}$, where $\mathbf{E}_{\tau}^{\top} \mathbf{E}_{\sigma}=\mathbf{U} \Sigma \mathbf{V}^{\top}$ is the singular value decomposition of $\mathbf{E}_{\tau}^{\top} \mathbf{E}_{\sigma}[4,23]$.

A query's embedding is defined as the average of the embeddings of its tokens, an effective practice for short texts [8, 42, 66, 72]. We denote with $\mathbf{v}_{\mathrm{S}_{i}}, \mathbf{v}_{\mathrm{T}_{j}}$ both $\in \mathbb{R}^{1 \times d}$, the embeddings of a source query (from $Q_{\mathrm{S}}$ ) and of a target query from $\mathcal{P}_{\mathrm{T}}$, respectively. Then, an element $\omega_{i j}$ from the cosine similarity matrix $\Omega \in \mathbb{R}^{s \times\left|\mathcal{P}_{\mathrm{T}}\right|}$ between the embeddings of source and valid target queries is given by $\omega_{i j}=$ $\left(\mathbf{v}_{\mathrm{S}_{i}} \mathbf{W} \mathbf{v}_{\mathrm{T}_{j}}^{\top}\right) /\left(\left\|\mathbf{v}_{\mathrm{S}_{i}} \mathbf{W}\right\|_{2}\left\|\mathbf{v}_{\mathrm{T}_{j}}\right\|_{2}\right)$. Note that the cosine similarities are computed after projecting the embeddings of the source domain to the target domain using the transformation matrix $\mathbf{W}$.

In theory, we can directly use $\omega_{i j}$ to determine the $k$ most similar target queries to the source query, thus providing a one-to-many mapping. However, in practice when conducting translations based on cross-lingual word embeddings, this may result in the presence of "hubs", i.e. target words or queries that are similar to unrealistically many different source words, a development that reduces the performance of translation $[18,57]$. Smith et al. mitigate this effect by using an inverted softmax ranking, described next [57].

Given $q_{i}$ in the source language, its translation is determined by finding candidate target queries $q_{j}^{\prime}$ that maximize the probability defined by

$$
P_{j \rightarrow i}=\frac{\exp \left(\eta \omega_{i j}\right)}{\alpha_{j} \sum_{z=1}^{s} \exp \left(\eta \omega_{i z}\right)},
$$

where $\alpha_{j}$ is a normalization factor that ensures $P_{j \rightarrow i}$ is a probability, and $s$ is the number of source queries in the vocabulary. The inverted softmax estimates the probability $P_{j \rightarrow i}$ that a candidate target query translates back to the source query, rather than

\footnotetext{
${ }^{7}$ The embeddings were obtained from gi thub.com/facebookresearch/fastText
} 
the other way around, $P_{i \rightarrow j}[18,57]$. If a target query is a hub, then the denominator in Eq. 5 will be large, preventing this target query from being selected. The parameter $\eta$ is learned by maximizing the log probability over the alignment dictionary $(\sigma \rightarrow \tau)$, i.e., $\operatorname{argmax}_{\eta} \sum_{\text {pairs } i j} \ln \left(P_{j \rightarrow i}\right)$. The top- $k$ queries from $\mathcal{P}_{\mathrm{T}}$ with the highest pairing probability $\left(P_{j \rightarrow i}\right)$ are then selected as possible translations of the source query $q_{i}$. Finally, we compute the semantic (cosine) similarity score $\Theta_{\mathrm{s}}$ between the source query $q_{i}$ and the target query $q_{j}$ using $\Theta_{\mathrm{s}}\left(q_{i}, q_{j}\right)=\left(\mathbf{e}_{q_{i}} \mathbf{W} \mathbf{e}_{q_{j}}^{\top}\right) /\left(\left\|\mathbf{e}_{q_{i}} \mathbf{W}\right\|_{2}\left\|\mathbf{e}_{q_{j}}\right\|_{2}\right)$, where $\mathbf{e}_{q_{i}}, \mathbf{e}_{q_{j}}$ are the embeddings of $q_{i}, q_{j}$, respectively. Our experiments report results for a variety of values of $k$.

If the language in the source and the target domain is the same, the previously described approach is not applicable. Given potential differences in querying preferences across different countries, some of the source queries, $Q_{S}$, may not be present in the pool of candidate target queries, $\mathcal{P}_{\mathrm{T}}$. Therefore, we use cosine similarity to map each source query to the $k$ most similar target ones using the common word embedding space for the shared language.

Temporal correlation similarity $\left(\Theta_{\mathrm{c}}\right)$. We compute the Pearson correlation between the frequency time series of the source and target queries over a fixed period (set to 5 years in our experiments). Since the flu season may be offset in the target domain with respect to the source domain, we computed the maximum correlation between these two frequency time series using a shifting window of $\pm \xi$ weeks. The range of possible values for $\xi$ is determined based on the seasonal offset between the source and target countries (see Section 4). Given a source query, $q_{i}$, and a target query, $q_{j}$ which is a member of a mapping set $\mathcal{T}_{i}$ (consisting of $k \geq 1$ queries from $\left.\mathcal{P}_{\mathrm{T}}\right)$, and their associated daily search frequencies, $\mathbf{x}_{i}(t)$ and $\mathbf{x}_{j}(t)$ respectively, the temporal correlation similarity, $\Theta_{c}$, is given by

$$
\Theta_{\mathrm{c}}\left(q_{i}, q_{j}\right)=\rho\left(\mathbf{x}_{i}(t), \mathbf{x}_{j}\left(t+l_{i j}\right)\right),
$$

where $\rho\left(x_{i}(t), x_{j}\left(t+l_{i j}\right)\right)$ denotes the optimal Pearson correlation coefficient between $\mathbf{x}_{i}, \mathbf{x}_{j}$ within the shifting window. Note that the optimal window is independently computed for each target query in $\mathcal{T}_{i}$, and thus optimal shifts may vary.

3.2.3 Step $3-$ Weighting target queries. In the previous steps, we have established that a source query $q_{i}$, which has received a regression weight $w_{i}$, is mapped to a set, $\mathcal{T}_{i}$, of $k \geq 1$ queries in the target domain. If $k=1$, then we can directly assign $w_{i}$ to the single target query. If $k>1$, then the source query's weight, $w_{i}$, should be distributed across these $k$ mapping target queries. To perform this, we have considered two alternatives:

- Uniform. We divide the source query weight, $w_{i}$, by the number of queries $q_{j}^{\prime}$ in $\mathcal{T}_{i}$, and assign each query in $\mathcal{T}_{i}$ a weight equal to $w_{j}^{\prime}=w_{i} / k$.

- Non-uniform. The $k$ target query weights are determined based on each target query's similarity score $\Theta_{i j}, j \in\{2, \ldots, k\}$, with the source query (see Eq. 3). More specifically, a target weight $w_{j}^{\prime}$ is defined as $w_{j}^{\prime}=w_{i} \Theta_{i j^{\prime}} / \sum_{q_{j}^{\prime} \in \mathcal{T}_{i}} \Theta_{i j^{\prime}}$.

To obtain a baseline performance estimate, we randomly shuffle the established query mappings in Step 2, and then transfer the source weights to $k$ target queries using the uniform approach. We repeat this process multiple times and report the mean performance of these randomized transfer learning models.

\section{EXPERIMENTS}

We deploy the proposed transfer learning framework to estimate ILI rates in three target countries without using any ground truth from these countries to supervise modeling. US is always set as the source country, while the target countries are FR, ES and AU. We assess the performance of the proposed model, comparing it to various baselines, and also provide a qualitative analysis, aiming to interpret some of the intrinsic properties of our approach.

Settings. After applying the semantic filter (Eq. 2) to the pool of 34,121 US queries, 1,403 queries were retained. The applied evaluation protocol is as follows. We train a source model (US) using the first 5 flu seasons (2007-12). A flu season is conventionally defined as the 1-year long period from the first week in September to the last week of August in the next year. ${ }^{8}$ Prior to applying elastic net, we maintain search queries that have $\mathrm{a} \geq .3$ Pearson correlation with the US ILI rates (these queries may vary per training fold). We then transfer the model to FR, ES, and AU and test it in the following flu season (2012-13). Then, we move our training data window to include the 2012-13 flu season and remove the first flu season (200708), and test in the following season (2013-14), so that we still have 5 flu seasons to train. We repeat this process until we have tested on the last flu season in our data set (2015-16), evaluating performance 4 times in total. The window size $(\xi)$ used for identifying optimal correlations between the frequency time series of the source and target queries (see Section 3 ) is set to \pm 6 weeks for FR and ES. The window is the same for $\mathrm{AU}$, although prior to applying it, the query frequency time series are shifted by 6 months to account for the seasonal difference in the northern and southern hemispheres. For the one-to- $k$ mapping from a source to a set of target queries, we explore sizes up to $k=5$ (values $>5$ did not yield any different insights). We measure the performance of transferred models by comparing our estimates with their national public health estimates, using Pearson correlation $(r)$, mean absolute error (MAE), and root mean squared error (RMSE). Regression errors are computed after reverting inferences back to their corresponding non standardized values.

Baseline models. To demonstrate the effectiveness of our transfer learning framework, we compare it with four baseline models:

- Random. After determining the mapping between source and target queries, the pairs (one-to- $k$ ) are randomly permuted. The source query weight is uniformly distributed across the mapped $k$ target queries. We repeat this process 2,000 times and report the average inference performance. This random assignment of query weights provides a possibly worst case baseline.

- Transfer component analysis (TCA). TCA is a transfer learning approach that aims to learn transfer components across source and target domains in a reproducing kernel Hilbert space using maximum mean discrepancy [48]. After we map source to target queries, TCA is applied to source and target query frequencies.

- Unsupervised query selection based on semantic similarity. We apply a semantic filter (described in Eq. 2) to remove queries that are irrelevant to the flu topic. The term pairs \{'grippe',

\footnotetext{
${ }^{8}$ Note that for AU this may result into including the end of a flu season and the beginning of the next in training and test folds.
} 
Table 2: Performance estimates for the US $\rightarrow$ FR transfer learning task. Different values of $\gamma$ determine how queries are mapped from the source to the target domain $(\gamma=1$ : semantic similarity only, $\gamma=0$ : temporal correlation only, $\gamma \in(0,1)$ : joint similarity score). Numbers in parentheses represent the standard deviation of the error. The best performance among all transfer learning models is denoted in bold. The best performance among models under a common $\gamma$ value is underlined. Only the best random mapping performance $(R)$ is enumerated per choice of $\gamma$. The last two rows show the performance of the baseline models.

\begin{tabular}{|c|c|c|c|c|c|c|c|c|c|c|c|c|c|c|c|c|c|}
\hline \multirow{2}{*}{ Mapping } & \multirow{2}{*}{$k$} & \multirow{2}{*}{$\mathbf{w}$} & \multicolumn{3}{|c|}{$09 / 2012-09 / 2013$} & \multicolumn{3}{|c|}{$09 / 2013-09 / 2014$} & \multicolumn{3}{|c|}{$09 / 2014-09 / 2015$} & \multicolumn{3}{|c|}{$09 / 2015-09 / 2016$} & \multicolumn{3}{|c|}{ Average } \\
\hline & & & $\mathbf{r}$ & MAE & RMSE & $\mathbf{r}$ & MAE & RMSE & $\mathbf{r}$ & MAE & RMSE & $\mathbf{r}$ & MAE & RMSE & $\mathbf{r}$ & MAE & RMSE \\
\hline \multirow{10}{*}{$\gamma=0$} & 1 & - & .797 & 78.905 & 136.098 & .789 & 59.584 & 93.752 & .900 & 56.107 & 92.324 & .855 & 51.533 & 78.073 & $.835(.045)$ & $61.532(10.429)$ & $100.062(21.690)$ \\
\hline & 2 & $\mathrm{U}$ & .803 & 80.044 & 137.247 & .794 & 59.961 & 94.853 & .890 & 58.372 & 96.282 & .843 & 55.532 & 84.438 & $.833(.038)$ & $\overline{63.477(9.696)}$ & $\overline{103.205(20.179)}$ \\
\hline & 3 & $\mathrm{U}$ & .802 & 79.010 & 135.905 & .796 & 59.750 & 95.350 & .896 & 57.241 & 94.451 & .844 & 57.306 & 86.588 & $.834(.040)$ & $63.327(9.111)$ & $103.073(19.260)$ \\
\hline & 4 & $\mathrm{U}$ & .798 & 79.077 & 135.892 & .795 & 59.529 & 95.295 & .895 & 58.380 & 95.852 & .834 & 59.729 & 90.180 & $.830(.040)$ & $64.179(8.617)$ & $104.305(18.370)$ \\
\hline & 5 & $\mathrm{U}$ & .799 & 78.881 & 135.743 & .794 & 58.508 & 95.036 & .893 & 58.439 & 96.988 & .829 & 60.075 & 91.182 & $.829(.040)$ & $63.976(8.630)$ & $104.737(18.023)$ \\
\hline & 2 & $\mathrm{NU}$ & .803 & 80.012 & 137.180 & .794 & 59.971 & 94.869 & .891 & 58.360 & 96.268 & .843 & 55.502 & 84.399 & $.833(.038)$ & $63.461(9.689)$ & $103.179(20.159)$ \\
\hline & 3 & NU & .802 & 78.999 & 135.881 & .796 & 59.763 & 95.360 & .896 & 57.244 & 94.453 & .844 & 57.271 & 86.538 & $.834(.040)$ & $63.319(9.110)$ & $103.058(19.259)$ \\
\hline & 4 & $\mathrm{NU}$ & .799 & 79.068 & 135.875 & .795 & 59.519 & 95.278 & .895 & 58.367 & 95.834 & .834 & 59.676 & 90.106 & $.830(.040)$ & $64.157(8.623)$ & $104.273(18.381)$ \\
\hline & 5 & NU & .799 & 78.868 & 135.725 & .794 & 58.499 & 95.015 & .893 & 58.434 & 96.972 & .829 & 60.029 & 91.110 & $.829(.040)$ & $63.957(8.632)$ & $104.706(18.033)$ \\
\hline & 1 & $\mathrm{R}$ & .771 & 125.422 & 152.275 & .731 & 93.122 & 105.769 & .807 & 138.579 & 158.000 & .825 & 102.972 & 113.607 & $.783(.036)$ & $115.024(17.943)$ & $132.413(22.982)$ \\
\hline \multirow{14}{*}{$\gamma=1$} & 1 & - & .964 & 51.885 & 77.728 & .928 & 24.373 & 35.801 & .974 & 51.623 & 69.254 & .917 & 75.416 & 92.946 & $.946(.024)$ & $50.824(18.071)$ & $68.932(20.927)$ \\
\hline & 2 & $\mathrm{U}$ & .967 & 41.298 & 68.164 & .939 & 22.993 & 33.287 & .973 & 62.869 & 81.119 & .924 & 84.469 & 102.422 & $.951(.020)$ & $52.907(23.049)$ & 71.248 (25.099) \\
\hline & 3 & $\mathrm{U}$ & .967 & 39.789 & 67.336 & .947 & 21.219 & 30.446 & .972 & 58.654 & 79.471 & .933 & 76.235 & & $.955(.016)$ & $48.974(20.564)$ & \\
\hline & 4 & $\mathrm{U}$ & .965 & 40.120 & 65.882 & .947 & 24.037 & 33.095 & .970 & 63.290 & 85.390 & .939 & 77.601 & 93.301 & .955 & 38) & 4) \\
\hline & 5 & $\mathrm{U}$ & .965 & 37.632 & 61.217 & .952 & 26.136 & 35.651 & .972 & 66.825 & 90.248 & .943 & 78.479 & 93.855 & $.958(.011)$ & 52.26 & 70 \\
\hline & 2 & NU & .968 & 41.272 & 68.016 & .939 & 22.925 & 33.213 & .973 & 61.971 & 80.280 & .924 & 83.058 & 101.160 & $.951(.020)$ & 52.306 & 70.667 \\
\hline & 3 & $\mathrm{NU}$ & .967 & 39.665 & 66.933 & .948 & 21.189 & 30.378 & .973 & 58.568 & 79.476 & .933 & 75.661 & 92.917 & $.955(.016)$ & $48.770(20.388)$ & $67.426(23.280)$ \\
\hline & 4 & NU & .966 & 39.754 & 65.480 & .948 & 23.794 & 32.767 & .971 & 62.957 & 85.275 & .939 & 76.868 & 92.866 & $.956(.013)$ & $50.843(20.486)$ & $69.097(23.236)$ \\
\hline & 5 & $\mathrm{NU}$ & .966 & 37.295 & 60.749 & .952 & 25.925 & 35.383 & .972 & 66.890 & 90.583 & .943 & 77.969 & 93.647 & $.958(.012)$ & 52.020 & 70.091 \\
\hline & 3 & $\mathrm{R}$ & .891 & 83.535 & 113.537 & .890 & 79.396 & 86.904 & .949 & 116.532 & 124.478 & .922 & 109.746 & 119.219 & $.913(.024)$ & 84) & 111. \\
\hline & 2 & $\mathrm{C}$ & .968 & 39.972 & 65.695 & .941 & 21.639 & 31.190 & .974 & 59.103 & 77.964 & .926 & 78.798 & 97.444 & $.952(.019)$ & $49.878(21.313)$ & $68.073(24.117)$ \\
\hline & 3 & $\mathrm{C}$ & .967 & 38.062 & 64.349 & .949 & 20.408 & 29.002 & .973 & 56.188 & 77.822 & .933 & 72.492 & 90.289 & $.956(.016)$ & $\underline{46.788(19.501)}$ & $65.365(22.911)$ \\
\hline & 4 & $\mathrm{C}$ & .965 & 38.225 & 63.063 & .949 & 22.869 & 31.161 & .971 & 60.623 & 83.764 & .938 & 73.644 & 90.367 & & & \\
\hline & 5 & $\mathrm{C}$ & .966 & 35.827 & 58.820 & .953 & 24.940 & 33.619 & .973 & 63.562 & 87.764 & .942 & 74.547 & 90.793 & $.958(.012)$ & $49.719(20.094)$ & $67.749(23.325)$ \\
\hline \multirow{10}{*}{$\gamma_{\mathrm{opt}}=.5$} & 1 & - & .968 & 33.475 & 53.775 & .951 & 22.615 & 34.416 & .973 & 34.793 & 58.007 & .944 & 45.324 & 62.417 & $.959(.012)$ & $\underline{34.052(8.043)}$ & $52.153(10.687)$ \\
\hline & 2 & $\mathrm{U}$ & .959 & 37.461 & 60.529 & .939 & & 38.056 & .967 & 43.197 & & .930 & 54.504 & 74.766 & $.949(.015)$ & & \\
\hline & 3 & $\mathrm{U}$ & .954 & 38.786 & 63.909 & .939 & 26.390 & 39.771 & .968 & 44.241 & 71.312 & .931 & 61.182 & 81.592 & .948( & 42.6 & 04.14 \\
\hline & 4 & $\mathrm{U}$ & .948 & 41.150 & 69.125 & .934 & 29.553 & 43.996 & .966 & 47.021 & 74.662 & .932 & 62.330 & 82.811 & $.945(.014)$ & $45.014(11.810)$ & $67.649(14.498)$ \\
\hline & 5 & $\mathrm{U}$ & .945 & 41.936 & 71.322 & .925 & 30.387 & 46.164 & .963 & 46.108 & 75.703 & .931 & 61.750 & 82.670 & $.941(.015)$ & $45.045(11.233)$ & $68.965(13.772)$ \\
\hline & 2 & $\mathrm{NU}$ & .959 & 37.414 & 60.456 & .939 & 24.881 & 38.036 & .967 & 43.118 & 69.763 & .930 & 54.329 & 74.599 & $.949(.015)$ & $39.936(10.610)$ & $60.714(14.045)$ \\
\hline & 3 & NU & .954 & 38.675 & & .940 & 26.423 & 39.789 & .968 & 44.452 & 71.495 & .931 & 61.147 & & & $42.674(12.495)$ & $64.169(15.428)$ \\
\hline & 4 & $\mathrm{NU}$ & .948 & 40.867 & 68.727 & .935 & 29.381 & 43.748 & .966 & 47.093 & 74.691 & .932 & 62.323 & 82.804 & $.945(.014)$ & $44.916(11.890)$ & $67.492(14.591)$ \\
\hline & 5 & NU & .946 & 41.610 & 70.892 & .926 & 30.201 & 45.863 & .963 & 46.192 & 75.685 & .931 & 61.788 & 82.685 & $.942(.015)$ & 44.948 (11.333) & $68.781(13.881)$ \\
\hline & 1 & $\mathrm{R}$ & .913 & 86.752 & 110.096 & .846 & 72.130 & 83.158 & .943 & 94.681 & 109.176 & .942 & 97.352 & 104.952 & $.911(.039)$ & $87.729(9.813)$ & $101.845(10.962)$ \\
\hline Uns & - & - & .936 & - & - & .870 & - & - & .947 & - & - & .910 & - & - & $.916(.030)$ & - & - \\
\hline Supervised & - & - & .977 & 27.331 & 50.643 & .979 & 23.665 & 33.994 & .992 & 34.345 & 62.803 & .987 & 15.011 & 21.956 & $.984(.006)$ & $25.088(6.970)$ & $42.349(15.595)$ \\
\hline
\end{tabular}

$k$ : number of target queries (1-to- $k$ mapping), w: weighting approach, $\mathbf{U}$ : uniform, NU: non-uniform, C: correlation, $\mathbf{R}$ : random

'fièvre'\}, \{'gripe', 'fiebre'\} and \{'flu', 'fever'\} are used to define this semantic filter in FR, ES and AU, respectively. Queries with $g \leq .5$ are filtered out and are not considered in our experiments. The mean weekly frequency of the retained queries is regarded as a proxy of the estimated ILI rates. These estimates are in different scale with the true ILI rates, thus we only report their Pearson correlation $(r)$.

- Supervised learning. We first apply a semantic filter (see point above) to the queries of each target country. We then train an elastic net, after maintaining only queries that have a moderate correlation with the ground truth ( $r \geq .3$ with the target values in the training data). This is inline with previously proposed, stateof-the-art supervised models for the task [38] and is considered as the top performance we could obtain, if we had access to ground truth in the target countries.

\subsection{Quantitative analysis}

Performance estimates are enumerated in Tables 2, 3, and 4 for each transfer learning task (US $\rightarrow$ FR, US $\rightarrow \mathrm{ES}$, US $\rightarrow \mathrm{AU}$ ). We first explored the extreme cases of $\gamma=0$ and $\gamma=1$ (Eq. 3) that result to using only temporal correlation or semantic similarity, respectively.

For $\gamma=0$, spurious queries could be included in the target domain's mappings. This is a result of the way the pool of target queries, $\mathcal{P}_{\mathrm{T}}$, was originally formed (see Section 2). Seasonal search queries, correlating with the occurrence of flu incidents in a population, are very likely to be selected as mappings, e.g. "symptoms flu" was mapped to "ski serre chevalier" in the US $\rightarrow$ FR task. Seasonal activities or expressions may change in time, and thus such queries are very unstable predictors. In fact, the best average performance we can obtain for $\gamma=0$ is considerably worse (MAEs of 61.532, 25.977 and 42.348 for FR, ES, and AU) than for alternative values. Setting $k=1$ provides the best results on average. In general, performance is not affected much by different choices of weighting (uniform, non-uniform) or the number of queries in a mapping $(k)$.

For $\gamma=1$, we obtain on average more accurate estimates than for $\gamma=0$. As a precursor to the joint similarity, we also introduce a correlation-based weighting scheme (denoted by "C"), which uses the optimal correlation between source and target queries (after 
Table 3: Performance estimates for US $\rightarrow$ ES transfer learning task. Please refer to Table 2's caption for further information.

\begin{tabular}{|c|c|c|c|c|c|c|c|c|c|c|c|c|c|c|c|c|c|}
\hline \multirow{2}{*}{ Mapping } & \multirow{2}{*}{$k$} & \multirow{2}{*}{$\mathbf{w}$} & \multicolumn{3}{|c|}{$09 / 2012-09 / 2013$} & \multicolumn{3}{|c|}{$09 / 2013-09 / 2014$} & \multicolumn{3}{|c|}{$09 / 2014-09 / 2015$} & \multicolumn{3}{|c|}{$09 / 2015-09 / 2016$} & \multicolumn{3}{|c|}{ Average } \\
\hline & & & $\mathbf{r}$ & MAE & RMSE & $\mathbf{r}$ & MAE & RMSE & $\mathbf{r}$ & MAE & RMSE & $\mathbf{r}$ & MAE & RMSE & $\mathbf{r}$ & MAE & RMSE \\
\hline \multirow{10}{*}{$\gamma=0$} & 1 & - & .808 & 25.068 & 41.104 & .807 & 25.789 & 42.137 & .843 & 29.221 & 47.360 & .791 & 25.134 & 38.497 & $.812(.019)$ & $26.303(1.708)$ & $42.275(3.222)$ \\
\hline & 2 & $\mathrm{U}$ & .799 & 25.589 & 42.631 & .843 & 23.850 & 39.092 & .844 & 30.069 & 48.120 & .821 & 24.470 & 36.902 & $.827(.019)$ & $25.994(2.434)$ & $41.686(4.240)$ \\
\hline & 3 & $\mathrm{U}$ & .795 & 25.756 & 42.883 & .840 & 23.669 & 38.934 & .843 & 29.509 & 48.189 & .813 & 24.989 & 37.713 & $.823(.020)$ & $25.981(2.169)$ & $41.930(4.088)$ \\
\hline & 4 & $\mathrm{U}$ & .783 & 26.504 & 43.662 & .835 & 23.671 & 39.207 & .844 & 29.850 & 48.335 & .809 & 25.715 & 38.745 & $.818(.024)$ & $26.435(2.226)$ & $42.487(3.884)$ \\
\hline & 5 & $\mathrm{U}$ & .783 & 26.579 & 43.391 & .840 & 23.605 & 38.861 & .842 & 30.336 & 48.800 & .806 & 26.586 & 39.843 & $.818(.025)$ & $26.776(2.388)$ & $42.724(3.892)$ \\
\hline & 2 & NU & .799 & 25.579 & 42.610 & .843 & 23.852 & 39.095 & .844 & 30.060 & 48.111 & .821 & 24.472 & 36.907 & $.827(.019)$ & $25.991(2.430)$ & $\underline{41.681(4.233)}$ \\
\hline & 3 & NU & .795 & 25.748 & 42.867 & .840 & 23.670 & 38.936 & .843 & 29.503 & 48.176 & .813 & 24.989 & 37.712 & $.823(.020)$ & $25.977(2.167)$ & $\overline{41.922(4.082)}$ \\
\hline & 4 & NU & .784 & 26.491 & 43.643 & .835 & 23.671 & 39.209 & .844 & 29.842 & 48.325 & .809 & 25.708 & 38.734 & $.818(.023)$ & $26.428(2.223)$ & $42.478(3.881)$ \\
\hline & 5 & NU & .783 & 26.567 & 43.380 & .840 & 23.605 & 38.866 & .842 & 30.324 & 48.785 & .806 & 26.575 & 39.826 & $.818(.025)$ & $26.768(2.384)$ & $42.714(3.887)$ \\
\hline & 3 & $\mathrm{R}$ & .830 & 40.548 & 46.584 & .903 & 36.241 & 40.718 & .846 & 53.929 & 61.098 & .813 & 45.762 & 49.637 & $.848(.034)$ & $44.120(6.591)$ & 49.509 (7.419) \\
\hline \multirow{14}{*}{$\gamma=1$} & 1 & - & .954 & 28.614 & 34.944 & .976 & 27.777 & 30.129 & .919 & 44.638 & 50.082 & .899 & 43.761 & 46.590 & $.937(.030)$ & $36.197(8.013)$ & $40.436(8.175)$ \\
\hline & 2 & $\mathrm{U}$ & .955 & 27.342 & 33.979 & .976 & 27.118 & 29.294 & .923 & 44.723 & 50.213 & .925 & 44.518 & 49.547 & $.945(.022)$ & $35.926(8.696)$ & $40.758(9.274)$ \\
\hline & 3 & $\mathrm{U}$ & .958 & 25.523 & 31.885 & .971 & 28.293 & 32.055 & .916 & 47.603 & 53.909 & .917 & 48.513 & 54.053 & $.941(.024)$ & $37.483(10.625)$ & 42.975 (11.006) \\
\hline & 4 & $\mathrm{U}$ & .960 & 25.316 & 31.623 & .973 & 27.998 & 31.797 & .918 & 46.862 & 53.458 & .918 & 47.443 & 52.823 & $.942(.025)$ & $36.905(10.294)$ & $42.425(10.718)$ \\
\hline & 5 & $\mathrm{U}$ & .957 & 24.445 & 30.821 & .975 & 27.169 & 30.959 & .917 & 45.775 & 52.620 & .914 & 45.854 & 51.505 & $.941(.026)$ & $35.811(10.050)$ & $41.476(10.594)$ \\
\hline & 2 & NU & .955 & 26.336 & 32.978 & .977 & 26.069 & 28.232 & .923 & 43.543 & 49.056 & .925 & 43.389 & 48.409 & $.945(.022)$ & $34.834(8.632)$ & $39.669(9.221)$ \\
\hline & 3 & NU & .958 & 25.532 & 31.879 & .971 & 28.327 & 32.076 & .917 & 47.471 & 53.737 & .917 & 48.356 & 53.908 & $.941(.024)$ & 37.422( & 10.923) \\
\hline & 4 & NU & .960 & 25.324 & 31.587 & .973 & 28.020 & 31.814 & .919 & 46.770 & 53.334 & .917 & 47.391 & 52.769 & $.942(.025)$ & $36.876(10.251)$ & $42.376(10.678)$ \\
\hline & 5 & NU & .958 & 24.432 & 30.759 & .975 & 27.197 & 30.990 & .917 & 45.778 & 52.576 & .915 & 45.951 & 51.574 & $.941(.026)$ & $35.839(10.073)$ & 41.475 (10.607) \\
\hline & 2 & $\mathrm{R}$ & .731 & 47.277 & 53.345 & .804 & 44.924 & 52.394 & .795 & 60.370 & 70.934 & .719 & 48.986 & 56.506 & $.762(.038)$ & $50.389(5.940)$ & $58.295(7.454)$ \\
\hline & 2 & $\mathrm{C}$ & .954 & 25.520 & 33.516 & .976 & 24.408 & 26.693 & .923 & 41.827 & 47.782 & .924 & 41.142 & 46.278 & $.944(.022)$ & $33.224(8.273)$ & $38.567(8.816)$ \\
\hline & 3 & $\mathrm{C}$ & .957 & 23.642 & 31.398 & .970 & 25.353 & 29.090 & .916 & 44.358 & 50.846 & .916 & 45.405 & 51.174 & $.940(.024)$ & $34.690(10.217)$ & $\overline{40.627(10.416)}$ \\
\hline & 4 & $\mathrm{C}$ & .960 & 23.339 & 30.912 & .973 & 24.900 & 28.709 & .919 & 43.431 & 50.236 & .918 & 44.297 & 49.873 & $.942(.025)$ & $33.992(9.892)$ & $39.933(10.153)$ \\
\hline & 5 & $\mathrm{C}$ & .957 & 24.137 & 30.513 & .974 & 26.555 & 30.466 & .917 & 44.598 & 51.464 & .915 & 45.662 & 51.359 & $.941(.026)$ & $35.238(9.936)$ & $40.950(10.461)$ \\
\hline \multirow{10}{*}{$\gamma_{\mathrm{opt}}=.2$} & 1 & - & .931 & 21.419 & 30.004 & .948 & 15.403 & 23.900 & .907 & 27.050 & 39.864 & .888 & 26.762 & 35.420 & $.918(.023)$ & $22.658(4.751)$ & $\underline{32.297(5.974)}$ \\
\hline & 2 & $\mathrm{U}$ & .926 & 21.433 & 29.944 & .941 & 17.334 & 25.525 & .899 & 30.166 & 43.243 & .877 & 30.662 & 40.272 & $.911(.025)$ & $\overline{24.899(5.705)}$ & $34.746(7.260)$ \\
\hline & 3 & $\mathrm{U}$ & .936 & 21.249 & 28.841 & .961 & 18.189 & 24.028 & .908 & 31.608 & 42.568 & .900 & 35.661 & 43.995 & $.926(.024)$ & 26.677 (7.186) & $34.858(8.608)$ \\
\hline & 4 & $\mathrm{U}$ & .945 & 21.016 & 28.161 & .965 & 18.720 & 23.647 & .917 & 32.235 & 41.483 & .910 & 37.141 & 44.448 & $.934(.022)$ & $27.278(7.654)$ & $34.435(8.742)$ \\
\hline & 5 & $\mathrm{U}$ & .946 & 20.977 & 28.041 & .967 & 18.727 & 23.321 & .910 & 33.330 & 43.018 & .903 & 36.846 & 44.547 & $.932(.026)$ & $27.470(7.760)$ & $34.732(9.219)$ \\
\hline & 2 & NU & .926 & 21.427 & 29.932 & .941 & 17.321 & 25.510 & .899 & 30.135 & 43.214 & .877 & 30.626 & 40.233 & $.911(.025)$ & 24.877 (5.694) & $34.723(7.251)$ \\
\hline & 3 & NU & .936 & 21.254 & 28.845 & .961 & 18.186 & 24.037 & .908 & 31.583 & 42.554 & .900 & 35.629 & 43.969 & $.926(.024)$ & $26.663(7.171)$ & $34.851(8.595)$ \\
\hline & 4 & NU & .945 & 21.023 & 28.158 & .965 & 18.739 & 23.682 & .917 & 32.241 & 41.509 & .910 & 37.128 & 44.438 & $.934(.022)$ & $27.283(7.643)$ & $34.447(8.734)$ \\
\hline & 5 & NU & .946 & 20.983 & 28.033 & .967 & 18.747 & 23.364 & .910 & 33.337 & 43.028 & .903 & 36.872 & 44.568 & $.932(.026)$ & $27.485(7.762)$ & $34.748(9.215)$ \\
\hline & 1 & $\mathrm{R}$ & .865 & 32.859 & 40.942 & .931 & 33.323 & 38.687 & .878 & 49.262 & 57.762 & .814 & 45.799 & 51.424 & $.872(.042)$ & $40.311(7.325)$ & $47.204(7.763)$ \\
\hline$\gamma=.5$ & 1 & - & .945 & 20.016 & 28.161 & .965 & 17.720 & 23.647 & .917 & 31.235 & 41.483 & .910 & 36.141 & 44.448 & $.934(.022)$ & $26.278(7.654)$ & $34.435(8.742)$ \\
\hline Un & - & - & .936 & - & - & .976 & - & - & .910 & - & - & .878 & - & - & $.925(.036)$ & - & - \\
\hline Supervised & - & - & .968 & 19.788 & 24.487 & .993 & 30.642 & 41.059 & .972 & 24.779 & 35.861 & .954 & 13.271 & 20.992 & $.971(.014)$ & $22.120(6.392)$ & $30.600(8.166)$ \\
\hline
\end{tabular}

$k$ : number of target queries (1-to- $k$ mapping), w: weighting approach, $\mathbf{U}$ : uniform, NU: non-uniform, C: correlation, $\mathbf{R}:$ random

deploying a shifting window) to determine the proportion of the source weight that will be allocated to the $k$ mapped queries. In countries that deploy a translation module based on bilingual word embeddings, the "C" scheme $(k=2$ or 3$)$ outperforms the other two (uniform, non-uniform). For the US $\rightarrow$ AU task, where high semantic similarity often means that very similar queries are being mapped to each other (given the common language), the optimal model is obtained for $k=1$, and thus, no further distribution of the weights is required. With or without the " $\mathrm{C}$ " weighting scheme, better performance is achieved compared to setting $\gamma=0$ (MAEs of 46.788/48.77, 33.224/34.834 and 34.509/30.275 for FR, ES, and AU).

The joint similarity scheme attempts to combine the positive attributes of semantic and correlation based similarities. To assess its potential contribution, we performed a grid search using 9 values of $\gamma$ (from .1 to .9), and presented the results for the best performing one $\left(\gamma_{\text {opt }}\right)$. For completeness, we also show results for the default choices of $\gamma=.5$ and $k=1$. Firstly, the application of the joint similarity leads to significant performance improvements in all tasks (MAEs of 34.052, 22.658 and 22.043 for FR, ES, and AU). Secondly, the best performing model consistently occurs for $k=1$, i.e. for one-to-one query mappings, where no weight redistribution is required. Finally, although results do not deviate much from the default settings of $\gamma=.5$ and $k=1$, there are discrepancies between the optimal $\gamma$ value for each task ( $\gamma_{\mathrm{opt}}=.5, .2$ and .9 for FR, ES, and $\mathrm{AU})$. One possible explanation may be that this is an artefact of the intrinsic characteristics (size, semantic/temporal similarities) of the pool of candidate target queries used for each task (see Section 4.2).

Better performance is always obtained (in terms of MAE and RMSE) compared to the random mapping allocation baseline ("R"), the best performance estimates of which per $\gamma$ value are provided. The same holds for TCA, which performs even worse than random (results are omitted). One explanation for this is that TCA fails to capture the time series structure of this particular data set, an essential property for producing a meaningful solution. Furthermore, the optimal models (joint similarity) outperform the unsupervised baseline in terms of correlation, the only metric which is relevant in this case. Finally, compared to the fully supervised elastic net, the transfer learning unsupervised approach reaches to a comparable performance, which is worse by $23.15 \%, 5.55 \%$, and $17.5 \%$ (in terms of RMSE), for FR, ES, and AU, respectively.

Fig. 2 plots the time series of a selection of these estimates, including the ones of the best performing models, in comparison to the ground truth, for each target country. We can see how estimates become significantly better when the joint similarity is 
Table 4: Performance estimates for the US $\rightarrow$ AU transfer learning task. Please refer to Table 2's caption for further information.

\begin{tabular}{|c|c|c|c|c|c|c|c|c|c|c|c|c|c|c|c|c|c|}
\hline \multirow{2}{*}{ Mapping } & \multirow{2}{*}{$k$} & \multirow{2}{*}{$\mathbf{w}$} & \multicolumn{3}{|c|}{$09 / 2012-09 / 2013$} & \multicolumn{3}{|c|}{$09 / 2013-09 / 2014$} & \multicolumn{3}{|c|}{$09 / 2014-09 / 2015$} & \multicolumn{3}{|c|}{$09 / 2015-09 / 2016$} & \multicolumn{3}{|c|}{ Average } \\
\hline & & & $\mathbf{r}$ & MAE & RMSE & $\mathbf{r}$ & MAE & RMSE & $\mathbf{r}$ & MAE & RMSE & $r$ & MAE & RMSE & $\mathbf{r}$ & MAE & RMSE \\
\hline \multirow{10}{*}{$\gamma=0$} & 1 & - & .704 & 38.804 & 50.140 & .677 & 39.151 & 48.508 & .630 & 51.412 & 65.215 & .787 & 40.025 & 57.421 & $.700(.056)$ & $42.348(5.359)$ & $55.321(6.830)$ \\
\hline & 2 & $\mathrm{U}$ & .622 & 41.824 & 55.943 & .663 & 41.708 & 50.752 & .633 & 52.017 & 66.448 & .763 & 40.557 & 59.312 & $.670(.055)$ & $44.027(4.734)$ & $\overline{58.114(5.873)}$ \\
\hline & 3 & $\mathrm{U}$ & .621 & 42.263 & 56.819 & .669 & 42.900 & 51.487 & .631 & 53.041 & 67.754 & .769 & 41.330 & 59.468 & $.672(.058)$ & $44.883(4.840)$ & $58.882(6.055)$ \\
\hline & 4 & $\mathrm{U}$ & .607 & 42.040 & 56.755 & .669 & 42.501 & 51.008 & .634 & 51.868 & 66.404 & .759 & 40.287 & 58.660 & $.667(.056)$ & $44.174(4.611)$ & $58.207(5.678)$ \\
\hline & 5 & $\mathrm{U}$ & .600 & 41.900 & 56.618 & .671 & 41.950 & 49.692 & .647 & 50.958 & 64.744 & .761 & 40.899 & 58.979 & $.670(.058)$ & $43.927(4.164)$ & $57.508(5.561)$ \\
\hline & 2 & $\mathrm{NU}$ & .623 & 41.886 & 55.947 & .663 & 41.642 & 50.818 & .633 & 52.068 & 66.590 & .763 & 40.617 & 59.384 & $.670(.055)$ & $44.053(4.747)$ & $58.185(5.908)$ \\
\hline & 3 & $\mathrm{NU}$ & .620 & 42.263 & 56.812 & .668 & 42.857 & 51.533 & .631 & 53.062 & 67.852 & .769 & 41.373 & 59.540 & $.672(.058)$ & $44.889(4.845)$ & $58.934(6.081)$ \\
\hline & 4 & $\mathrm{NU}$ & .607 & 42.031 & 56.745 & .669 & 42.466 & 51.039 & .634 & 51.909 & 66.504 & .759 & 40.343 & 58.732 & $.667(.056)$ & $44.187(4.621)$ & $58.255(5.708)$ \\
\hline & 5 & $\mathrm{NU}$ & .600 & 41.885 & 56.601 & .671 & 41.928 & 49.723 & .647 & 51.011 & 64.844 & .761 & 40.935 & 59.032 & $.670(.058)$ & $43.940(4.186)$ & $57.550(5.589)$ \\
\hline & 1 & $\mathrm{R}$ & .653 & 60.835 & 71.392 & .710 & 52.090 & 62.045 & .628 & 67.895 & 78.856 & .738 & 69.695 & 75.320 & $.683(.043)$ & $62.629(7.069)$ & $71.903(6.468)$ \\
\hline \multirow{14}{*}{$\gamma=1$} & 1 & - & .916 & 23.447 & 26.436 & .871 & 13.994 & 18.129 & .902 & 35.315 & 42.126 & .971 & 48.344 & 50.617 & $.915(.035)$ & $30.275(13.143)$ & $34.327(13.150)$ \\
\hline & 2 & $\mathrm{U}$ & .900 & 28.828 & 33.029 & .880 & 18.583 & 22.656 & .925 & 39.274 & 45.149 & .989 & 59.174 & 60.026 & $.923(.040)$ & 36.465 & $40.215(14$ \\
\hline & 3 & $\mathrm{U}$ & .896 & 30.804 & 35.148 & .881 & 19.492 & 23.743 & .938 & 36.748 & 42.294 & .990 & 57.829 & 58.516 & $.926(.041)$ & $36.218(14.216)$ & 39.925 (12.999) \\
\hline & 4 & $\mathrm{U}$ & .889 & 30.876 & 35.549 & .872 & 21.475 & 26.089 & .935 & 37.484 & 42.966 & .994 & 57.871 & 58.397 & $.922(.047)$ & $36.926(13.636)$ & $40.750(12.180)$ \\
\hline & 5 & $\mathrm{U}$ & .882 & 31.248 & 35.738 & .868 & 21.320 & 25.883 & .936 & 37.615 & 43.059 & .992 & 58.773 & 59.318 & $.919(.047)$ & 37.239 & 41.000 \\
\hline & 2 & $\mathrm{NU}$ & .902 & 28.789 & 32.947 & .880 & 18.497 & 22.565 & .925 & 39.278 & 45.150 & .989 & 59.007 & 59.861 & $.924(.039)$ & $36.393(15.287)$ & 40.131 (14.347) \\
\hline & 3 & $\mathrm{NU}$ & .897 & 30.805 & 35.137 & .882 & 19.510 & 23.775 & .938 & 36.973 & 42.482 & .990 & 57.779 & 58.462 & $.927(.041)$ & $36.267(14.193)$ & $39.964(12.978)$ \\
\hline & 4 & $\mathrm{NU}$ & .890 & 30.839 & 35.484 & .873 & 21.367 & 25.986 & .936 & 37.554 & 42.999 & .994 & 57.825 & 58.354 & $.923(.046)$ & $36.896(13.655)$ & $40.706(12.205)$ \\
\hline & 5 & $\mathrm{NU}$ & .884 & 31.217 & 35.678 & .870 & 21.261 & 25.830 & .936 & 37.609 & 43.019 & .992 & 58.770 & 59.309 & $.920(.047)$ & $37.214(14.022)$ & 40.959 (12.603) \\
\hline & 1 & $\mathrm{R}$ & .825 & 58.539 & 60.310 & .793 & 42.200 & 46.818 & .890 & 55.940 & 61.462 & .963 & 65.023 & 66.924 & $.868(.064)$ & $55.426(8.491)$ & $58.878(7.627)$ \\
\hline & 2 & $\mathrm{C}$ & .905 & 27.444 & 31.356 & .881 & 17.547 & 21.520 & .925 & 37.373 & 43.387 & .989 & 58.318 & 59.229 & $.925(.040)$ & $35.171(15.399)$ & $38.873(14.510)$ \\
\hline & 3 & $\mathrm{C}$ & .900 & 28.802 & 32.701 & .882 & 18.039 & 22.091 & .939 & 34.534 & 40.310 & .990 & 56.660 & 57.516 & $.928(.041)$ & $34.509(14.381)$ & $38.154(13.316)$ \\
\hline & 4 & $\mathrm{C}$ & .894 & 28.643 & 32.867 & .874 & 19.505 & 23.747 & .938 & 34.613 & 40.360 & .994 & 56.309 & 57.011 & $.925(.045)$ & $34.768(13.828)$ & 38.496 (12.579) \\
\hline & 5 & $\mathrm{C}$ & .888 & 29.149 & 33.118 & .870 & 19.259 & 23.507 & .939 & 34.622 & 40.252 & .992 & 57.220 & 57.962 & $.922(.047)$ & $35.063(14.211)$ & $38.710(12.993)$ \\
\hline \multirow{10}{*}{$\gamma_{\mathrm{opt}}=.9$} & 1 & - & .922 & 11.997 & 14.986 & .879 & 15.084 & 18.011 & .898 & 24.898 & 31.110 & .985 & 36.191 & 38.271 & $.921(.039)$ & $22.043(9.649)$ & $25.594(9.796)$ \\
\hline & 2 & $\mathrm{U}$ & .892 & 16.642 & 19.922 & .881 & 15.719 & 19.009 & .923 & 23.858 & 30.280 & .988 & 39.919 & 41.175 & $.921(.041)$ & $\overline{24.034(9.895)}$ & $\overline{27.596(9.282)}$ \\
\hline & 3 & $\mathrm{U}$ & .890 & 18.641 & 22.543 & .876 & 18.391 & 21.453 & .930 & 23.965 & 29.934 & .989 & 41.232 & 42.249 & $.921(.043)$ & $25.557(9.510)$ & $29.045(8.549)$ \\
\hline & 4 & $\mathrm{U}$ & .883 & 19.078 & 23.494 & .866 & 19.766 & 22.757 & .928 & 23.691 & 29.686 & .991 & 40.159 & 41.138 & $.917(.047)$ & $25.673(8.721)$ & $29.269(7.590)$ \\
\hline & 5 & $\mathrm{U}$ & .875 & 20.091 & 24.960 & .862 & 18.791 & 21.614 & .933 & 23.474 & 29.474 & .991 & 41.433 & 42.483 & $.915(.050)$ & $25.947(9.288)$ & $29.633(8.171)$ \\
\hline & 2 & $\mathrm{NU}$ & .894 & 16.565 & 19.826 & .882 & 15.679 & 18.961 & .923 & 23.830 & 30.226 & .988 & 39.809 & 41.071 & $.922(.040)$ & $23.971(9.873)$ & $27.521(9.270)$ \\
\hline & 3 & $\mathrm{NU}$ & .892 & 18.588 & 22.457 & .877 & 18.312 & 21.353 & .930 & 23.995 & 29.967 & .989 & 41.230 & 42.245 & $.922(.042)$ & $25.531(9.534)$ & $29.005(8.589)$ \\
\hline & 4 & $\mathrm{NU}$ & .885 & 19.043 & 23.410 & .867 & 19.639 & 22.621 & .929 & 23.690 & 29.673 & .991 & 40.229 & 41.204 & $.918(.047)$ & $25.650(8.781)$ & $29.227(7.665)$ \\
\hline & 5 & $\mathrm{NU}$ & .877 & 19.983 & 24.795 & .864 & 18.716 & 21.530 & .933 & 23.414 & 29.390 & .991 & 41.416 & 42.462 & $.916(.049)$ & $25.882(9.318)$ & $29.544(8.210)$ \\
\hline & 1 & $\mathrm{R}$ & .844 & 47.859 & 50.120 & .817 & 37.727 & 40.926 & .900 & 54.008 & 59.263 & .940 & 55.980 & 59.071 & $.875(.047)$ & $48.893(7.254)$ & $52.345(7.791)$ \\
\hline$\gamma=.5$ & 1 & - & .871 & 18.642 & 23.367 & .848 & 17.735 & 20.735 & .873 & 27.140 & 32.733 & .930 & 39.651 & 43.484 & $.880(.298)$ & $25.792(8.982)$ & $30.080(9.208)$ \\
\hline Unsupe & - & - & .815 & - & - & .810 & - & - & .881 & - & - & .942 & - & - & $.862(.054)$ & - & - \\
\hline Supervised & - & - & .891 & 19.353 & 25.297 & .865 & 22.048 & 25.200 & .939 & 18.658 & 22.473 & .971 & 11.255 & 14.159 & $.916(.041)$ & $17.829(4.001)$ & $21.782(4.545)$ \\
\hline
\end{tabular}

$k$ : number of target queries (1-to- $k$ mapping), w: weighting approach, $\mathbf{U}$ : uniform, NU: non-uniform, C: correlation, $\mathbf{R}:$ random

used versus its extremes. The transferred models can very often estimate the peak of the flu season accurately. This includes the time of occurrence as well as its intensity. Notably, ILI rates in the target countries differ in terms of scale compared to ones of the source, but the proposed models are capable of capturing different scales effortlessly, providing further evidence about the user search behavior similarities among different countries (Section 3.1). At the same time, most models show some inaccuracies, especially during the time periods with very moderate flu circulation (e.g. summer).

\subsection{Qualitative analysis}

A fair criticism for the proposed framework is that in a practical scenario the optimal values for $\gamma$ and $k$ cannot be validated. However, we have already demonstrated that the default settings of $\gamma=.5$ and $k=1$ provide very satisfactory performance in all our case studies. Fig. 3 looks further into this, depicting performance estimates (MAE) for different values of $\gamma$. As discussed previously, optimal $\gamma$ values differ per target country. Interestingly, all error trends are monotonically decreasing (as $\gamma$ increases) until they reach a minimum, and then begin to monotonically increase. We argue that $\gamma_{\text {opt }}$ reflects on the actual pool of candidate target queries $\left(\mathcal{P}_{\mathrm{T}}\right)$, although we have a small sample size to be able to empirically prove this. In our data, the average correlation over the average semantic similarity ratio between all source-target query pairs is equal to 1.143, .982 and 2.261, for the FR, ES, and AU tasks respectively. These ratios depend on characteristics of the target queries which we are not controlling for in our approach. They do correlate with the respective optimal $\gamma$ values $(.5, .2$, and .9$)$, an insight that can be used to make a more informed choice of $\gamma$ in future applications of the proposed framework.

Table 5 lists the top- 5 query mappings that were the most impactful in the ILI estimates on average during the 10 weeks with the lowest and greatest MAEs (for the optimal transfer models). Impact is determined by the percentage of an estimated ILI rate that is contributed by a query (frequency $\times$ weight / estimated ILI rate). The identified pairs during the weeks with the lowest errors are topically coherent (about flu) and in many occasions are accurate translations from the source to the target language. On the other hand, pairs responsible for the largest errors include inaccurate translations that sometimes lead to an off-topic target query selection. For example, " 24 hour flu" is mapped to "grippe intestinale" (impact: 13.2\%), "child fever" to "sinusitis" (7.7\%), and "child temperature" to "warmer" (9.8\%). Nevertheless, it is encouraging

\footnotetext{
9 "Grippe intestinale" translates to "stomach flu" (formally "viral gastroenteritis").
} 

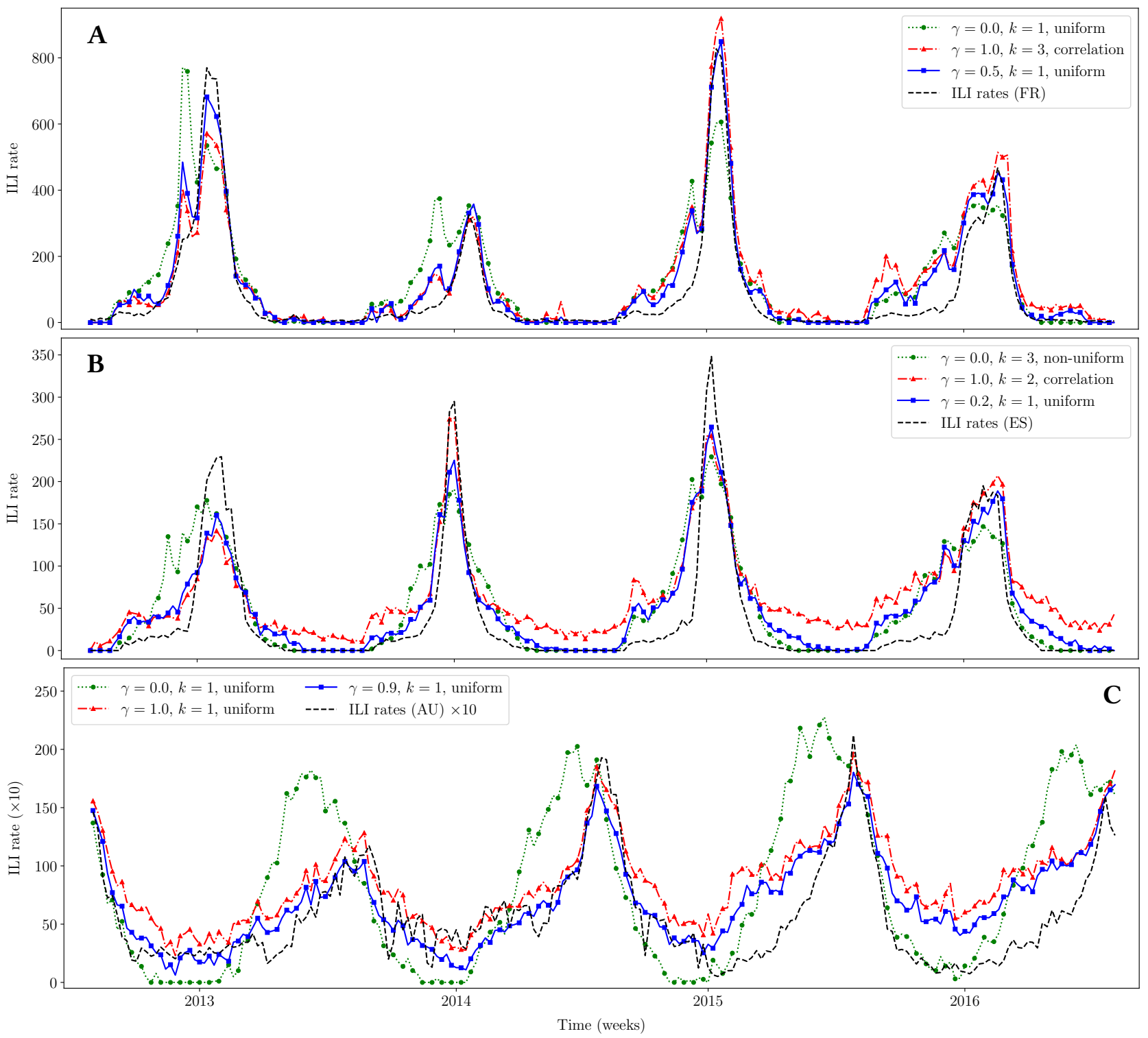

Figure 2: Comparison of transfer learning models for estimating ILI rates in France (A), Spain (B) and Australia (C) with the corresponding actual ILI rates obtained by health agencies in these countries.

that some of these mappings may have been avoided by carefully preprocessing the target query candidates to avoid spurious queries.

The optimal joint similarity transfer models do not improve by increasing the number of target queries $(k>1)$. An interpretation for that might be drawn by the fact that for $k=1$ at most $77.9 \%$ of the selected target queries are unique (at least $22.1 \%$ are repetitive selections). Hence, the method seems to be converging to a subset of queries already for $k=1$. As $k$ increases, the error increases monotonically. This might be due to the existence of various spurious queries in the feature space which are being introduced as additional mappings.

Finally, the choice of adding a non-negativity constraint to the regularized regression function for the source domain (Eq. 1), was also empirically justified. When it is removed, we can learn a more accurate source model for the US, but the MAE on the target countries increases on average by $20.6 \%, 21.6 \%$, and $20.5 \%$ for FR, ES, and AU respectively. This confirms our original assumption that transferring negative weights is a harder task, and thus, error-prone.

\section{RELATED WORK}

The fundamental properties of transfer learning have been thoroughly discussed in relevant literature [6, 7, 40, 49, 59, 65]. In contrast to traditional machine learning methods, which assume that the training and test data belong to the same domain, i.e. they are drawn from the same feature space and distribution, transfer learning aims to improve the learning function in a target domain by 
Table 5: Top-5 target queries (with source mappings) in terms of mean ILI estimate impact (\%) in the 10 weeks with the lowest and greatest MAE (all test periods), for all target countries (TC), based on their respective optimal transfer learning models.

\begin{tabular}{|c|c|c|}
\hline TC & Mappings during accurate estimates & Mappings during inaccurate estimates \\
\hline$\overline{\text { FR }}$ & $\begin{array}{l}\text { flu incubation period } \rightarrow \text { grippe durée (10.9), cough fever } \rightarrow \text { la toux (6.3), how to } \\
\text { treat flu } \rightarrow \text { comment soigner une grippe }(6) \text {, fever flu } \rightarrow \text { fièvre de la grippe }(5.47) \text {, } \\
\text { flu treatment } \rightarrow \text { traitement de la grippe (4.95) }\end{array}$ & $\begin{array}{l}24 \text { hour flu } \rightarrow \text { grippe intestinale }(13.24) \text {, influenza a treatment } \rightarrow \text { grippe traite- } \\
\text { ment }(8.07) \text {, remedies for colds } \rightarrow \text { rhume de cerveau }(6.75) \text {, child tempera- } \\
\text { ture } \rightarrow \text { température du corps }(6.37) \text {, child fever } \rightarrow \text { fièvre adulte }(6.04)\end{array}$ \\
\hline$\overline{\mathbf{E S}}$ & $\begin{array}{l}\text { symptoms of flu } \rightarrow \text { symptômes grippe }(9.04) \text {, fever flu } \rightarrow \text { con gripe (7.49), cough } \\
\text { fever } \rightarrow \text { la tos }(6.34) \text {, flu incubation period } \rightarrow \text { cuanto dura una gripe }(5.19) \text {, how } \\
\text { to treat a fever } \rightarrow \text { para bajar la fiebre }(5.03)\end{array}$ & $\begin{array}{l}\text { mucinez for kids } \rightarrow \text { tratmiento de la grippe (20.76), child fever } \rightarrow \text { sinusitis (7.76), } \\
\text { influenza a treatment } \rightarrow \text { con gripe (7.02), symptoms pneumonia } \rightarrow \text { bronquitis } \\
(6.04) \text {, child temperature } \rightarrow \text { temperatura corporal }(5.62)\end{array}$ \\
\hline$\overline{\mathrm{AU}}$ & $\begin{array}{l}\text { treatment for the flu } \rightarrow \text { flu treatment }(9.85) \text {, cough fever } \rightarrow \text { cough and fever } \\
(8.05), \text { flu type } \rightarrow \text { influenza type }(5.37) \text {, symptoms of flu } \rightarrow \text { symptoms of flu } \\
\text { (5.11), flu incubation period } \rightarrow \text { flu incubation period (5.03) }\end{array}$ & $\begin{array}{l}24 \text { hour flu } \rightarrow \text { flu duration }(11.51) \text {, child temperature } \rightarrow \text { warmer }(9.77) \text {, how } \\
\text { to treat a fever } \rightarrow \text { have a fever }(6.94) \text {, tamiflu and breastfeeding } \rightarrow \text { flu while } \\
\text { pregnant }(6.81) \text {, robitussin } \mathrm{cf} \rightarrow \text { colds }(5.18)\end{array}$ \\
\hline
\end{tabular}

transferring knowledge from a related, source domain. This concept has been successfully applied to various tasks, including text classification [14, 16, 22, 48], part of speech tagging [10, 28], machine translation [20,29], and image classification [19, 30, 71].

In this work, we present a statistical framework for transferring a disease surveillance model from a source country, where supervised learning is applicable, to a target country, where no ground truth is available. We formulate it as a cross-lingual transductive regression task [49], which poses the following challenges: (a) ground truth is not available in the target domain, and (b) features (queries) may not belong in the same feature space due to linguistic or cultural differences. Due to (a), multi-task learning models, such as this solution for ILI [72], cannot be used because they still require partial ground truth from the target domain to capture the relationship between the different tasks [13]. To solve (b), a few studies have attempted to learn a mapping of both source and target languages to the same space [27, 55, 57, 64]. For example, Prettenhofer and Stein used unlabeled documents along with a word translation oracle to automatically induce task-specific, cross-lingual correspondences for cross-lingual text classification [55]. In this paper, we used crosslingual word embeddings to align different languages [57].

Methods have also been proposed for reducing the distance between the source and target features [48, 70]. For example, Pan et al. proposed TCA to learn transfer components across source and target domains in a reproducing kernel Hilbert space using maximum mean discrepancy [48]. Zhou et al. constructed a sparse feature transformation matrix based on compressive sensing theory to

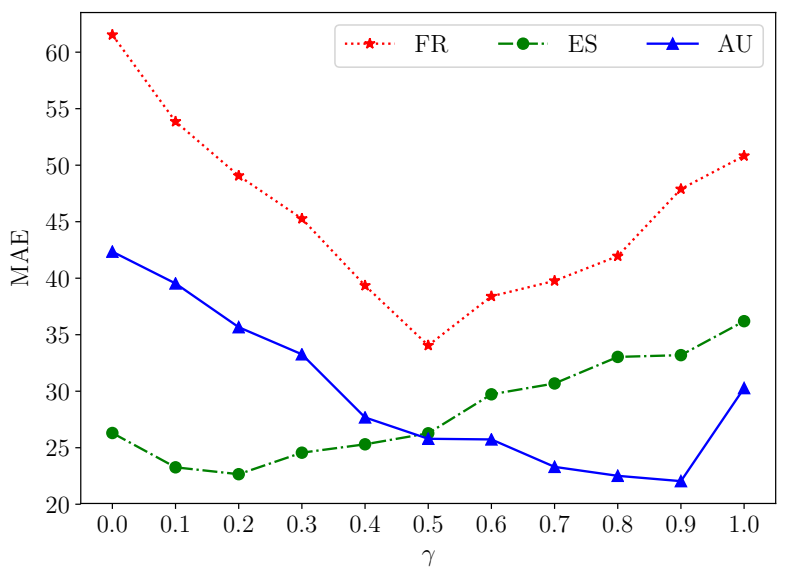

Figure 3: MAE under different $\gamma$ values for the transfer learning models for FR, ES, and AU $(k=1)$. map the weight vector of classifiers learned from the source domain to the target domain [70]. However, their tasks are very different from the regression task studied in this paper. These models were not able to capture efficiently the time series structure in our data.

Finally, the topic of disease modelling, and in particular of ILI, from online user-generated content has been extensively studied in the literature. The vast majority of methods proposed supervised solutions, using social media or search engine data together with disease rates from an established health authority [15, 21, 32, 33, $35,38,50,52,53,67]$. A few unsupervised methods have also been attempted, but they showcased moderate accuracy in terms of correlation [31,51]. Our approach is able to provide accurate estimates without using any ground truth in the target locations.

\section{CONCLUSIONS}

Prior work on estimating disease rates from online user-generated content relies heavily on supervised learning models. Such models require ground truth data which is usually provided by public health organizations. Syndromic surveillance data, however, is either sparse or absent from locations with a poor healthcare infrastructure. This is somewhat ironic as it is often stated that web-based approaches hold considerable promise for regions that lack an established health surveillance system. This paper proposes a transfer learning framework as a potential solution to this problem. We leverage semantic and temporal relationships to map a supervised model from a source to a target location. We show that we can obtain a satisfactory performance $(r>.92$ on average) that does not deviate much from a fully supervised model $(\leq 21.6 \%$ increase in RMSE), without using any ground truth from the target domain.

There is a number of avenues for future work. It is highly desirable to perform a study where the target country is from a low or middle income region. However, such a study is complicated, since the lack of ground truth data does not allow the performance to be quantified. Nevertheless, a qualitative study that demonstrated ILI estimates that followed an expected seasonal pattern would be of value. Our experiments on regions with ground truth data allowed us to investigate parameters $k$ and $\gamma$, i.e. the choice for the one-to- $k$ mapping and the relative weight assigned to the semantic and temporal similarities. Our analysis indicated that a one-to-one $(k=1)$ mapping performed best on average, and that the optimal $\gamma$ differed per target country. Although we attempted to justify both outcomes, further experiments on other regions are needed to understand the effect of these parameters better. 


\section{ACKNOWLEDGMENTS}

This work has been supported by the EPSRC grants EP/K031953/1 and EP/R00529X/1. The authors would like to acknowledge RCGP, PHE and SISSS for providing anonymized and aggregated syndromic surveillance data. Special thanks to Amparo Larrauri (SISSS) and Richard Pebody (PHE). We also thank Simon Moura and Peter Hayes for providing constructive feedback.

\section{REFERENCES}

[1] C. Alicino, N. L. Bragazzi, V. Faccio, D. Amicizia, D. Panatto, R. Gasparini, G. Icardi, and A. Orsi. 2015. Assessing Ebola-related Web Search Behaviour: Insights and Implications from an Analytical Study of Google Trends-based Query Volumes. Infectious Diseases of Poverty 4, 54 (2015), 1-13.

[2] W. Ammar, G. Mulcaire, Y. Tsvetkov, G. Lample, C. Dyer, and N. A. Smith 2016. Massively Multilingual Word Embeddings. arXiv Preprint (2016), arXiv: 1602.01925 .

[3] H. K. Andreassen, M. M. Bujnowska-Fedak, C. E. Chronaki, R. C. Dumitru, I Pudule, S. Santana, H. Voss, and R. Wynn. 2007. European Citizens' Use of E-health Services: A Study of Seven Countries. BMC Public Health 7, 53 (2007).

[4] M. Artetxe, G. Labaka, and E. Agirre. 2016. Learning Principled Bilingual Mappings of Word Embeddings while Preserving Monolingual Invariance. In Proceedings of the 2016 Conference on Empirical Methods in Natural Language Processing. Association for Computational Linguistics, 2289-2294.

[5] M. Artetxe, G. Labaka, E. Agirre, and K. Cho. 2018. Unsupervised Neural Machine Translation. In Proceedings of the 6th International Conference on Learning Representations.

[6] S. Ben-David, J. Blitzer, K. Crammer, A. Kulesza, F. Pereira, and J. W. Vaughan. 2010. A Theory of Learning from Different Domains. Machine Learning 79, 1-2 (2010), 151-175

[7] S. Ben-David, J. Blitzer, K. Crammer, and F. Pereira. 2007. Analysis of Representations for Domain Adaptation. In Proceedings of Advances in Neural Information Processing Systems 19. 137-144.

[8] A. Benton, R. Arora, and M. Dredze. 2016. Learning Multiview Embeddings of Twitter Users. In Proceedings of the 54th Annual Meeting of the Association for Computational Linguistics, Vol. 2. 14-19.

[9] M. Biggerstaff, M. Johansson, D. Alper, L. C. Brooks, P. Chakraborty, D. C. Farrow, S. Hyun, S. Kandula, C. McGowan, N. Ramakrishnan, et al. 2018. Results from the Second Year of A Collaborative Effort to Forecast Influenza Seasons in the United States. Epidemics 24 (2018), 26-33.

[10] J. Blitzer, R. McDonald, and F. Pereira. 2006. Domain Adaptation with Structural Correspondence Learning. In Proceedings of the 2006 Conference on Empirical Methods in Natural Language Processing. 120-128.

[11] G. Boivin, I. Hardy, G. Tellier, and J. Maziade. 2000. Predicting Influenza Infections during Epidemics with Use of a Clinical Case Definition. Clinical Infectious Diseases 31, 5 (2000), 1166-1169.

[12] P. Bojanowski, E. Grave, A. Joulin, and T. Mikolov. 2017. Enriching Word Vectors with Subword Information. Transactions of the Association of Computational Linguistics 5, 1 (2017), 135-146.

[13] R. Caruana. 1998. Multitask Learning. In Learning to Learn. Springer, 95-133.

[14] M. Chen, K. Q. Weinberger, and J. Blitzer. 2011. Co-Training for Domain Adaptation. In Proceedings of Advances in Neural Information Processing Systems 24 2456-2464.

[15] A. Culotta. 2010. Towards Detecting Influenza Epidemics by Analyzing Twitter Messages. In Proceedings of the 1st Workshop on Social Media Analytics. 115-122.

[16] W. Dai, G. Xue, Q. Yang, and Y. Yu. 2007. Transferring Naive Bayes Classifiers for Text Classification. In Proceedings of the 22nd International Conference on Artificial Intelligence. 540-545.

[17] M. De Choudhury, M. Gamon, S. Counts, and E. Horvitz. 2013. Predicting Depression via Social Media. In Proceedings of the 7th International AAAI Conference on Weblogs and Social Media. 128-137.

[18] G. Dinu, A. Lazaridou, and M. Baroni. 2014. Improving Zero-shot Learning by Mitigating the Hubness Problem. arXiv Preprint (2014), arXiv:1412.6568.

[19] L. Duan, D. Xu, and I. W. Tsang. 2012. Learning with Augmented Features for Heterogeneous Domain Adaptation. In Proceedings of the 29th International Coference on International Conference on Machine Learning. 667-674.

[20] G. Foster, C. Goutte, and R. Kuhn. 2010. Discriminative Instance Weighting for Domain Adaptation in Statistical Machine Translation. In Proceedings of the 2010 Conference on Empirical Methods in Natural Language Processing. 451-459.

[21] J. Ginsberg, M. H. Mohebbi, R. S. Patel, L. Brammer, M. S. Smolinski, and L. Brilliant. 2009. Detecting Influenza Epidemics using Search Engine Query Data. Nature 457, 7232 (2009), 1012-1014.

[22] X. Glorot, A. Bordes, and Y. Bengio. 2011. Domain Adaptation for Large-Scale Sentiment Classification: A Deep Learning Approach. In Proceedings of the 28th International Conference on Machine Learning. 513-520.
[23] G. H. Golub and C. Reinsch. 1970. Singular Value Decomposition and Least Squares Solutions. Numerische Mathematik 14, 5 (1970), 403-420.

[24] J. Gomide, A. Veloso, W. M. Jr., V. Almeida, F. Benevenuto, F. Ferraz, and M. Teixeira. 2011. Dengue Surveillance Based on a Computational Model of Spatiotemporal Locality of Twitter. In Proceedings of the 3rd International Web Science Conference. Article 3, 3:1-3:8 pages.

[25] O. Higgins, J. Sixsmith, M. M. Barry, and C. Domegan. 2011. A Literature Review on Health Information Seeking Behaviour on the Web: A Health Consumer and Health Professional Perspective. Technical Report.

[26] A. E. Hoerl and R. W. Kennard. 1970. Ridge Regression: Biased Estimation for Nonorthogonal Problems. Technometrics 12 (1970), 55-67.

[27] J.-T. Huang, J. Li, D. Yu, L. Deng, and Y. Gong. 2013. Cross-Language Knowledge Transfer using Multilingual Deep Neural Network with Shared Hidden Layers. In Proceedings of the 2013 IEEE International Conference on Acoustics, Speech and Signal Processing. 7304-7308.

[28] J. Jiang and C. Zhai. 2007. Instance Weighting for Domain Adaptation in NLP. In Proceedings of the 45th Annual Meeting of the Association of Computational Linguistics. 264-271.

[29] P. Koehn and J. Schroeder. 2007. Experiments in Domain Adaptation for Statistical Machine Translation. In Proceedings of the 2nd Workshop on Statistical Machine Translation. 224-227.

[30] B. Kulis, K. Saenko, and T. Darrell. 2011. What you Saw is not What you Get: Domain Adaptation using Asymmetric Kernel Transforms. In Proceedings of the IEEE Conference on Computer Vision and Pattern Recognition. 1785-1792.

[31] A. Lamb, M. J. Paul, and M. Dredze. 2013. Separating Fact from Fear: Tracking Flu Infections on Twitter. In Proceedings of the 2013 Conference of the North American Chapter of the Association for Computational Linguistics: Human Language Technologies. 789-795.

[32] V. Lampos and N. Cristianini. 2010. Tracking the flu pandemic by monitoring the social Web. In Proceedings of the 2nd International Workshop on Cognitive Information Processing. IEEE Press, 411-416.

[33] V. Lampos and N. Cristianini. 2012. Nowcasting Events from the Social Web with Statistical Learning. ACM Transactions on Intelligent Systems and Technology 3, 4 (2012), 1-22.

[34] V. Lampos, T. De Bie, and N. Cristianini. 2010. Flu Detector - Tracking Epidemics on Twitter. In Proceedings of the 2010 European Conference on Machine Learning and Knowledge Discovery in Databases: Part III (ECML PKDD'10). Springer, 599602

[35] V. Lampos, A. C. Miller, S. Crossan, and C. Stefansen. 2015. Advances in nowcasting influenza-like illness rates using search query logs. Scientific Reports 5 , 12760 (2015)

[36] V. Lampos, D. Preoţiuc-Pietro, and T. Cohn. 2013. A user-centric model of voting intention from Social Media. In Proceedings of the 51st Annual Meeting of the Association for Computational Linguistics (Volume 1: Long Papers). Association for Computational Linguistics, 993-1003.

[37] V. Lampos, E. Yom-Tov, R. Pebody, and I. J. Cox. 2015. Assessing the Impact of a Health Intervention via User-Generated Internet Content. Data Mining and Knowledge Discovery 29, 5 (2015), 1434-1457.

[38] V. Lampos, B. Zou, and I. J. Cox. 2017. Enhancing Feature Selection Using Word Embeddings: The Case of Flu Surveillance. In Proceedings of the 26th International Conference on World Wide Web. 695-704.

[39] O. Levy, A. Søgaard, and Y. Goldberg. 2017. A Strong Baseline for Learning Cross-Lingual Word Embeddings from Sentence Alignments. In Proceedings of the 15th Conference of the European Chapter of the Association for Computational Linguistics, Vol. 1. 765-774.

[40] Y. Mansour, M. Mohri, and A. Rostamizadeh. 2009. Domain Adaptation: Learning Bounds and Algorithms. arXiv Preprint (2009), arXiv:0902.3430.

[41] T. Mikolov, Q. V. Le, and I. Sutskever. 2013. Exploiting Similarities among Languages for Machine Translation. arXiv Preprint (2013), arXiv:1309.4168.

[42] T. Mikolov, I. Sutskever, K. Chen, G. S. Corrado, and J. Dean. 2013. Distributed Representations of Words and Phrases and Their Compositionality. In Proceedings of Advances in Neural Information Processing Systems 26. 3111-3119.

[43] G. J Milinovich, G. M Williams, A. C. A. Clements, and W. Hu. 2014. Internetbased Surveillance Systems for Monitoring Emerging Infectious Diseases. The Lancet Infectious Diseases 14, 2 (2014), 160-168.

[44] A. Mogadala and A. Rettinger. 2016. Bilingual Word Embeddings from Parallel and Non-parallel Corpora for Cross-language Text Classification. In Proceedings of the 2016 Conference of the North American Chapter of the Association for Computational Linguistics: Human Language Technologies. 692-702.

[45] M. Mohebbi, D. Vanderkam, J. Kodysh, R. Schonberger, H. Choi, and S. Kumar. 2011. Google Correlate Whitepaper. https://www.google.com/trends/correlate/ whitepaper.pdf.

[46] A. S. Monto, S. Gravenstein, M. Elliott, M. Colopy, and J. Schweinle. 2000. Clinical Signs and Symptoms Predicting Influenza Infection. Archives of Internet Medicine $160,21(2000), 3243-3247$.

[47] N. Mrkšić, I. Vulić, D. Ó Séaghdha, I. Leviant, R. Reichart, M. Gašić, A. Korhonen, and S. Young. 2017. Semantic Specialisation of Distributional Word Vector Spaces using Monolingual and Cross-lingual Constraints. arXiv Preprint (2017), 
arXiv: 1706.00374

[48] S. J. Pan, I. W. Tsang, J. T. Kwok, and Q. Yang. 2009. Domain Adaptation via Transfer Component Analysis. In Proceedings of the 21st International foint Conference on Artificial Intelligence. 1187-1192.

[49] S. J. Pan and Q. Yang. 2010. A Survey on Transfer Learning. IEEE Transactions on Knowledge and Data Engineering 22, 10 (2010), 1345-1359.

[50] M. J. Paul and M. Dredze. 2011. You Are What You Tweet: Analysing Twitter for Public Health. In Proceedings of the 5th International AAAI Conference on Weblogs and Social Media. 265-272.

[51] Michael J. Paul and Mark Dredze. 2014. Discovering Health Topics in Social Media Using Topic Models. PLOS One 9, 8 (2014), e103408.

[52] M. J. Paul, M. Dredze, and D. Broniatowski. 2014. Twitter Improves Influenza Forecasting. PLOS Currents 6 (2014).

[53] P. M. Polgreen, Y. Chen, D. M. Pennock, F. D. Nelson, and R. A. Weinstein. 2008 Using Internet Searches for Influenza Surveillance. Clinical Infectious Diseases 47, 11 (2008), 1443-1448.

[54] D. Preoţiuc-Pietro, S. Volkova, V. Lampos, Y. Bachrach, and N. Aletras. 2015 Studying User Income through Language, Behaviour and Affect in Social Media. PLOS ONE 10, 9 (2015), e0138717.

[55] P. Prettenhofer and B. Stein. 2010. Cross-language Text Classification using Structural Correspondence Learning. In Proceedings of the 48th Annual Meeting of the Association for Computational Linguistics. 1118-1127.

[56] S. Ruder. 2017. A Survey of Cross-Lingual Embedding Models. arXiv Preprint (2017), arXiv:1706.04902

[57] S. L. Smith, D. H. P. Turban, S. Hamblin, and N. Y. Hammerla. 2016. Offline Bilingual Word Vectors, Orthogonal Transformations and the Inverted Softmax arXiv Preprint (2016), arXiv:1702.03859.

[58] R. Tibshirani. 1996. Regression Shrinkage and Selection via the Lasso. fournal of the Royal Statistical Society: Series B (Statistical Methodology) 58, 1 (1996), 267-288.

[59] L. Torrey and J. Shavlik. 2009. Transfer Learning. Handbook of Research on Machine Learning Applications and Trends: Algorithms, Methods, and Techniques: Algorithms, Methods, and Techniques (2009), 242.

[60] I. Vulić and M.-F. Moens. 2015. Bilingual Word Embeddings from Non-Paralle Document-Aligned Data Applied to Bilingual Lexicon Induction. In Proceedings of the 53rd Annual Meeting of the Association for Computational Linguistics and the 7th International foint Conference on Natural Language Processing, Vol. 2. 719-725.

[61] I. Vulić and M.-F. Moens. 2015. Monolingual and Cross-lingual Information Retrieval Models based on (Bilingual) Word Embeddings. In Proceedings of the 38th International ACM SIGIR Conference on Research and Development in Information Retrieval. 363-372.

[62] I. Vulić and M.-F. Moens. 2016. Bilingual Distributed Word Representations from Document-Aligned Comparable Data. Journal of Artificial Intelligence Research
55 (2016), 953-994.

[63] M. Wagner, V. Lampos, I. J. Cox, and R. Pebody. 2018. The Added Value of Online User-generated Content in Traditional Methods for Influenza Surveillance. Scientific Reports 8, 13963 (2018).

[64] X. Wan. 2009. Co-training for Cross-lingual Sentiment Classification. In Proceedings of the Joint Conference of the 47th Annual Meeting of the Association of Computational Linguistics and the 4th International foint Conference on Natural Language Processing of the Asian Federation of Natural Language Processing. 235-243.

[65] K. Weiss, T. M. Khoshgoftaar, and D. Wang. 2016. A Survey of Transfer Learning. fournal of Big Data 3, 1 (2016).

[66] J. Xu, P. Wang, G. Tian, B. Xu, J. Zhao, F. Wang, and H. Hao. 2015. Short Text Clustering via Convolutional Neural Networks.. In Proceedings of the 2015 Conference of the North American Chapter of the Association for Computational Linguistics: Human Language Technologies. 62-69.

[67] S. Yang, M. Santillana, and S. C. Kou. 2015. Accurate Estimation of Influenza Epidemics using Google Search Data via ARGO. Proceedings of the National Academy of Sciences 112, 47 (2015), 14473-14478.

[68] M. Ybarra and M. Suman. 2008. Reasons, Assessments and Actions Taken: Sex and Age Differences in Uses of Internet Health Information. Health Education Research 23, 3 (2008), 512-521.

[69] P. Zhao and B. Yu. 2006. On Model Selection Consistency of Lasso. Fournal of Machine Learning Research 7 (2006), 2541-2563.

[70] J. T. Zhou, I. W. Tsang, S. J. Pan, and M. Tan. 2014. Heterogeneous Domain Adaptation for Multiple Classes. In Artificial Intelligence and Statistics. 10951103.

[71] Y. Zhu, Y. Chen, Z. Lu, S. J. Pan, G.-R. Xue, Y. Yu, and Q. Yang. 2011. Heterogeneous Transfer Learning for Image Classification. In Proceedings of the 25th AAAI Conference on Artificial Intelligence. 1304-1309.

[72] B. Zou, V. Lampos, and I. J. Cox. 2018. Multi-Task Learning Improves Disease Models from Web Search. In Proceedings of the 2018 World Wide Web Conference. International World Wide Web Conferences Steering Committee, 87-96.

[73] B. Zou, V. Lampos, R. Gorton, and I. J. Cox. 2016. On Infectious Intestinal Disease Surveillance using Social Media Content. In Proceedings of the 6th International Conference on Digital Health. ACM, 157-161.

[74] H. Zou and T. Hastie. 2005. Regularization and Variable Selection via the Elastic Net. Fournal of the Royal Statistical Society: Series B (Statistical Methodology) 67, 2 (2005), 301-320.

[75] W. Y. Zou, R. Socher, D. Cer, and C. D. Manning. 2013. Bilingual Word Embeddings for Phrase-based Machine Translation. In Proceedings of the 2013 Conference on Empirical Methods in Natural Language Processing. 1393-1398. 